\title{
Asymptotic Behaviors of Wronskians and Finite Asymptotic Expansions in the Real Domain. Part I: Scales of Regularly- or Rapidly-Varying Functions
}

\author{
Antonio Granata \\ Department of Mathematics and Computer Science \\ University of Calabria \\ Ponte Pietro Bucci, 87036 Rende (Cosenza), Italy \\ antoniogranata1973@gmail.com
}

\begin{abstract}
Keywords: Asymptotic Behaviors of Wronskians, Asymptotic Expansions in the Real Domain, Chebyshev Asymptotic Scales, Smoothly(Regularly)-Varying Functions of Higher Order, Rapidly-Varying Functions of Higher Order.
\end{abstract}

\begin{abstract}
In a previous series of papers we established a general theory of finite asymptotic expansions in the real domain for functions $f$ of one real variable sufficiently-regular on a deleted neighborhood of a point $x_{0} \in \overline{\mathbb{R}}$, a theory based on the use of a uniquely-determined linear differential operator $L$ associated to the given asymptotic scale and wherein various sets of asymptotic expansions are characterized by the convergence of improper integrals involving both the operator $L$ applied to $f$ and certain weight functions constructed by means of Wronskians of the given scale. Very special cases apart, Wronskians have quite complicated expressions and unrecognizable asymptotic behaviors; however in the present work, split in two parts, we highlight some approaches for determining the exact asymptotic behavior of a Wronskian when the involved functions are regularly- or rapidly-varying functions of higher order. This first Part contains: (i) some preliminary results on the asymptotic behavior of a determinant whose entries are asymptotically equivalent to the entries of a Vandermonde determinant; (ii) the fundamental results about the asymptotic behaviors of Wronskians involving scales of functions all of which are either regularly (or, more generally, smoothly) varying or rapidly varying of a suitable higher order. A lot of examples and applications to the theory of asymptotic expansions in the real domain are given.
\end{abstract}

\section{Introduction}

In a previously-published series of papers $[1,2,3,4,5]$ we have established a general theory of finite asymptotic expansions in the real domain for functions $f$ of one real variable sufficiently-regular on a deleted neighborhood of a point $x_{0} \in \overline{\mathbb{R}}$. The theory is based on the use of a uniquely-determined linear differential operator $L_{\phi_{1}, \ldots, \phi_{n}}$ associated to the given asymptotic scale:

$$
\phi_{1}(x) \gg \phi_{2}(x) \gg \cdots \gg \phi_{n}(x), x \rightarrow x_{0}^{-} .
$$

We gave conditions characterizing two special sets of expansions obtained from

$$
f(x)=a_{1} \phi_{1}(x)+\cdots+a_{n} \phi_{n}(x)+o\left(\phi_{n}(x)\right), x \rightarrow x_{0}^{-},
$$

by two different procedures of formal differentiation;these conditions are expressed via improper integrals involving both the quantity $L_{\phi_{1}, \ldots, \phi_{n}}[f(x)]$ and some functions defined as ratios of Wronskians of the $\phi_{i}$ 's; and these functions are the coefficients of two particular factorizations of $L_{\phi_{1}, \ldots, \phi_{n}}$ called "canonical factorizations". For instance one such condition is:

$$
\int_{T}^{\rightarrow x_{0}} \frac{W\left(\phi_{1}(t), \ldots, \phi_{n-1}(t)\right)}{W\left(\phi_{1}(t), \ldots, \phi_{n}(t)\right)} \cdot L_{\phi_{1}, \ldots, \phi_{n}}[f(t)] \mathrm{d} t \text { convergent. }
$$


Now for the special class of scales " $(\phi(x))^{\alpha_{1}} \gg \cdots \gg(\phi(x))^{\alpha_{n}}$ ” there is a simple formula giving a closed form of their Wronskians; it is reported in Lemma 5 below and has been used in [5; §15] to characterize useful expansions. But even for simple asymptotic scales such as

$$
x^{a_{1}} \cdot(\log x)^{b_{1}} \cdot \exp \left(c_{1} x\right) ; \ldots ; x^{a_{n}} \cdot(\log x)^{b_{n}} \cdot \exp \left(c_{n} x\right)
$$

and small values of $n \geq 3$ the Wronskians may have extremely cumbersome or even unreadable expressions. For instance, for $n=3$ we have the following explicit expressions, where $\ell_{k}(x)$ denotes the $k$-time iterated natural logarithm:

$$
\begin{aligned}
& W\left(\left(\ell_{1}(x)\right)^{b_{1}},\left(\ell_{2}(x)\right)^{b_{2}},\left(\ell_{3}(x)\right)^{b_{3}}\right)=-x^{-3}\left(\ell_{1}(x)\right)^{b_{1}-3}\left(\ell_{2}(x)\right)^{b_{2}-3}\left(\ell_{3}(x)\right)^{b_{3}-2} \times \\
& \times\left\{b_{1}^{2} b_{2}\left(\ell_{2}(x)\right)^{2}\left(\ell_{3}(x)\right)^{2}-b_{1}^{2} b_{3}\left(\ell_{2}(x)\right)^{2} \ell_{3}(x)+b_{1} b_{2}\left(1-b_{2}\right) \ell_{2}(x)\left(\ell_{3}(x)\right)^{2}+\right. \\
& \left.-b_{1} b_{3} \ell_{2}(x) \ell_{3}(x)+b_{1} b_{3}\left(b_{3}-1\right) \ell_{2}(x)+b_{2}^{2} b_{3} \ell_{3}(x)+b_{2} b_{3}\left(1-b_{3}\right)\right\}
\end{aligned}
$$

$$
\begin{aligned}
& W\left(x^{a_{1}}(\log x)^{b_{1}}, x^{a_{2}}(\log x)^{b_{2}}, x^{a_{3}}(\log x)^{b_{3}}\right)=-x^{a_{1}+a_{2}+a_{3}-3}(\log x)^{b_{1}+b_{2}+b_{3}} \times \\
& \times\left\{\left(-a_{1}^{2} a_{2}+a_{1} a_{2}^{2}+a_{1}^{2} a_{3}-a_{2}^{2} a_{3}-a_{1} a_{3}^{2}+a_{2} a_{3}^{2}\right)+\right. \\
& \begin{aligned}
(\log x)^{-1}\left(-2 a_{1} a_{2} b_{1}+a_{2}^{2} b_{1}+2 a_{1} a_{3} b_{1}-a_{3}^{2} b_{1}-a_{1}^{2} b_{2}+2 a_{1} a_{2} b_{2}-2 a_{2} a_{3} b_{2}+\right. \\
\left.\quad+a_{3}^{2} b_{2}+a_{1}^{2} b_{3}-a_{2}^{2} b_{3}-2 a_{1} a_{3} b_{3}+2 a_{2} a_{3} b_{3}\right)+ \\
(\log x)^{-2}\left(a_{2} b_{1}-a_{3} b_{1}-a_{2} b_{1}^{2}+a_{3} b_{1}^{2}-a_{1} b_{2}+a_{3} b_{2}-2 a_{1} b_{1} b_{2}+2 a_{2} b_{1} b_{2}+a_{1} b_{2}^{2}-a_{3} b_{2}^{2}+\right. \\
\left.\quad+a_{1} b_{3}-a_{2} b_{3}+2 a_{1} b_{1} b_{3}-2 a_{3} b_{1} b_{3}-2 a_{2} b_{2} b_{3}+2 a_{3} b_{2} b_{3}-a_{1} b_{3}^{2}+a_{2} b_{3}^{2}\right)+ \\
\left.(\log x)^{-3}\left(-b_{1}^{2} b_{2}+b_{1} b_{2}^{2}+b_{1}^{2} b_{3}-b_{2}^{2} b_{3}-b_{1} b_{3}^{2}+b_{2} b_{3}^{2}\right)\right\} ; \\
\quad \\
\quad W\left(x^{a_{1}} \cdot \exp \left(c_{1} x\right), x^{a_{2}} \cdot \exp \left(c_{2} x\right), x^{a_{3}} \cdot \exp \left(c_{3} x\right)\right)=e^{c_{1} x+c_{2} x+c_{3} x} x^{a_{1}+a_{2}+a_{3}} \times \\
\quad \times\left\{\left(c_{1}^{2} c_{2}-c_{1} c_{2}^{2}-c_{1}^{2} c_{3}+c_{2}^{2} c_{3}+c_{1} c_{3}^{2}-c_{2} c_{3}^{2}\right)+\right. \\
\quad+x^{-1}\left(a_{2} c_{1}^{2}-a_{3} c_{1}^{2}+2 a_{1} c_{1} c_{2}-2 a_{2} c_{1} c_{2}-a_{1} c_{2}^{2}+\right. \\
\left.\quad+a_{3} c_{2}^{2}-2 a_{1} c_{1} c_{3}+2 a_{3} c_{1} c_{3}+2 a_{2} c_{2} c_{3}-2 a_{3} c_{2} c_{3}+a_{1} c_{3}^{2}-a_{2} c_{3}^{2}\right)+ \\
\quad+x^{-2}\left(a_{2} c_{1}+2 a_{1} a_{2} c_{1}-a_{2}^{2} c_{1}-a_{3} c_{1}-2 a_{1} a_{3} c_{1}+a_{3}^{2} c_{1}-a_{1} c_{2}+a_{1}^{2} c_{2}-2 a_{1} a_{2} c_{2}+\right. \\
\left.\quad+a_{3} c_{2}+2 a_{2} a_{3} c_{2}-a_{3}^{2} c_{2}+a_{1} c_{3}-a_{1}^{2} c_{3}-a_{2} c_{3}+a_{2}^{2} c_{3}+2 a_{1} a_{3} c_{3}-2 a_{2} a_{3} c_{3}\right)+ \\
\left.\quad+x^{-3}\left(a_{1}^{2} a_{2}-a_{1} a_{2}^{2}-a_{1}^{2} a_{3}+a_{2}^{2} a_{3}+a_{1} a_{3}^{2}-a_{2} a_{3}^{2}\right)\right\} ;
\end{aligned}
\end{aligned}
$$

which are somehow readable but the corresponding Wronskians for $n=4$ have incredible two-page long expressions, as can be checked by a software like MATHEMATICA ${ }^{\circledR}$; and only for particular choices of the parameters $a_{i}, b_{i}, c_{i}$ the expressions reduce to handy objects. Hence practical applications of the theory are severely restricted. However in order to establish the validity or not of (3) the mere asymptotic behavior of the ratio of Wronskians may suffice, at least for absolute convergence; for instance from (4)-(6) we infer the following asymptotic relations as $x \rightarrow+\infty$ :

$$
\begin{gathered}
W\left(\left(\ell_{1}(x)\right)^{b_{1}},\left(\ell_{2}(x)\right)^{b_{2}},\left(\ell_{3}(x)\right)^{b_{3}}\right) \sim \\
\sim\left\{\begin{array}{lll}
-b_{1}^{2} b_{2} x^{-3}\left(\ell_{1}(x)\right)^{b_{1}-3} \cdot\left(\ell_{2}(x)\right)^{b_{2}-1} \cdot\left(\ell_{3}(x)\right)^{b_{3}} & \text { if } \quad b_{1}, b_{2} \neq 0 \\
b_{1}^{2} b_{3} x^{-3}\left(\ell_{1}(x)\right)^{b_{1}-3} \cdot\left(\ell_{2}(x)\right)^{b_{2}-1} \cdot\left(\ell_{3}(x)\right)^{b_{3}-1} & \text { if } \quad b_{2}=0 ; b_{1}, b_{3} \neq 0 \\
-b_{2}^{2} b_{3} x^{-3}\left(\ell_{1}(x)\right)^{b_{1}-3} \cdot\left(\ell_{2}(x)\right)^{b_{2}-3} \cdot\left(\ell_{3}(x)\right)^{b_{3}-1} & \text { if } \quad b_{1}=0 ; b_{2}, b_{3} \neq 0
\end{array}\right.
\end{gathered}
$$




$$
\begin{aligned}
& \left\{\begin{array}{l}
W\left(x^{a_{1}}(\log x)^{b_{1}}, x^{a_{2}}(\log x)^{b_{2}}, x^{a_{3}}(\log x)^{b_{3}}\right) \sim \\
\sim-\left(a_{3}-a_{2}\right)\left(a_{3}-a_{1}\right)\left(a_{2}-a_{1}\right) x^{a_{1}+a_{2}+a_{3}-3}(\log x)^{b_{1}+b_{2}+b_{3}}
\end{array}\right. \\
& \left\{\begin{array}{l}
W\left(x^{a_{1}} \cdot \exp \left(c_{1} x\right), x^{a_{2}} \cdot \exp \left(c_{2} x\right), x^{a_{3}} \cdot \exp \left(c_{3} x\right)\right) \sim \\
\sim-\left(c_{3}-c_{2}\right)\left(c_{3}-c_{1}\right)\left(c_{2}-c_{1}\right) x^{a_{1}+a_{2}+a_{3}} e^{c_{1} x+c_{2} x+c_{3} x}
\end{array}\right.
\end{aligned}
$$

in the cases wherein the $a_{i}$ 's in (8) and the $c_{i}$ 's in (9) are distinct numbers. Similar relations may be obtained for $n=4$ using MATHEMATICA ${ }^{\circledR}$. Our aim in this paper, split in two Parts, is to describe methods for finding out the asymptotic behaviors of the Wronskians of the most usual scales extending relations in (7)-(9) to any $n$ and to far more general scales. Our starting step consists in writing:

$$
\left|\begin{array}{ccc}
\phi_{1}(x) & \ldots & \phi_{n}(x) \\
\phi_{1}^{\prime}(x) & \ldots & \phi_{n}^{\prime}(x) \\
\ldots & \ldots & \ldots \\
\phi_{1}^{(n-1)}(x) & \ldots & \phi_{n}^{(n-1)}(x)
\end{array}\right|=\left(\prod_{i=1}^{n} \phi_{i}(x)\right) \cdot\left|\begin{array}{ccc}
1 & \ldots & 1 \\
\phi_{1}^{\prime}(x) / \phi_{1}(x) & \ldots & \phi_{n}^{\prime}(x) / \phi_{n}(x) \\
\phi_{1}^{\prime \prime}(x) / \phi_{1}(x) & \ldots & \phi_{n}^{\prime \prime}(x) / \phi_{n}(x) \\
\vdots & & \vdots \\
\phi_{1}^{(n-1)}(x) / \phi_{1}(x) & \ldots & \phi_{n}^{(n-1)}(x) / \phi_{n}(x)
\end{array}\right|,
$$

and then recalling that in various but related contexts the following remarkable asymptotic relations linking the ratios $\phi_{i}^{(k)}(x) / \phi_{i}(x)$ to $\phi_{i}^{\prime}(x) / \phi_{i}(x)$ are found, either

$$
\frac{\phi^{(k)}(x)}{\phi(x)}=\left(\frac{\phi^{\prime}(x)}{\phi(x)}\right)^{k}\left[\frac{(\alpha-1) \cdots(\alpha-k+1)}{\alpha^{k-1}}+o(1)\right], x \rightarrow+\infty, 1 \leq k \leq n,
$$

with a suitable real number $\alpha$, or

$$
\frac{\phi^{(k)}(x)}{\phi(x)} \sim\left(\frac{\phi^{\prime}(x)}{\phi(x)}\right)^{k}, x \rightarrow+\infty, 1 \leq k \leq n,
$$

for different classes of functions. Now, different approaches to (11) and (12) are found in the literature; sometimes in the form of sufficient conditions and always concerning infinitely-differentiable functions. For these reasons we decided to preliminarly systematize the "theories of higher-order types of asymptotic variation" for $C^{n}$-functions showing: (i) the equivalence of various approaches, (ii) the roles of a variety of "asymptotic functional equations", (iii) the output of algebraic operations done on the pertinent classes. This task has been settled in two semi-expository papers [6,7] which put us in a position to study our initial problem with a clear understanding of the assumptions about the involved classes of functions. For the time being just observe that most asymptotic scales found in applications when describing asymptotic properties at $+\infty$ of non-oscillatory differentiable functions are formed by products of compositions of a finite number of logarithms, powers and exponentials and that each such function satisfies (11) or (12).

Coming back to (10) the second step consists in replacing the ratios on the right by their principal parts as given in (11) and (12) and then proving that the latter determinant, a Vandermonde determinant with suitable elements, is asymptotically equivalent to the former. A complete treatment is rather long as different devices are required acccording as all the $\phi_{i}$ 's belong to the same class or if mixed types occur and this makes necessary the subdivision of the whole work in two Parts. The proofs of the main results in Part I, collected in Theorems 6, 9 and 10, require a certain amount of technical manipulations, but this is unavoidable due to the quite complicated expression of a Wronskian of order $n \geq 4$. Moreover, there are different types of results according to different grouping of the involved functions based on their growth-orders. We hope that the statements are sufficiently readable so as 
to make the results applicable as shown by the many examples given in $\S 5$. The main feature of our approach is that the three concepts of higher-order regular, smooth and rapid variation make the simple device in (10) work very well for a great number of often-encountered asymptotic scales.

The sections in the present Part I treat the following subjects:

$-\S 2$ contains a brief summary of the basic properties of various classes each with a specified type of asymptotic variation at $+\infty$, namely regular, smooth or rapid.

- $\S 3$ contains general results on the asymptotic behaviors of Vandermonde-like determinants i.e. determinants whose entries are functions such that their principal parts form a Vandermonde determinant.

- $\S 4$ contains the main results in the present Part I, namely the asymptotic behaviors of Wronskians of functions all of which are either regularly (smoothly) varying with different indexes of variation or rapidly varying.

$-\S 5$ contains many examples involving asymptotic scales.

- $\S 6$ contains applications to the analytic theory of finite asymptotic expansions inferred from convergence criteria for the integral in (3).

$-\S 7$ contains a few bibliographical notes.

In Part II we shall study the behaviors of Wronskians whose elements have different types of asymptotic variations.

General notations.

$-\mathbb{N}:=\{1,2, \ldots\} ; \mathbb{Z}:=\{0, \pm 1, \pm 2, \ldots\} ;$

$-\mathbb{R}:=$ real line; $\overline{\mathbb{R}} \equiv$ extended real line $:=\mathbb{R} \cup\{ \pm \infty\}$;

$-f \in A C^{0}(I) \equiv A C(I) \Leftrightarrow f$ is absolutely continuous on each compact subinterval of $I$;

$-f \in A C^{k}(I) \Leftrightarrow f^{(k)} \in A C(I)$;

- For $f \in A C^{k}(I)$ we write " $\lim _{x \rightarrow x_{0}} f^{(k+1)}(x)$ " meaning that $x$ runs through the points wherein $f^{(k+1)}$ exists as a finite number.

- Factorial powers:

$$
\alpha^{\underline{0}}:=1 ; \alpha^{\underline{1}}:=\alpha ; \alpha^{\underline{k}}:=\alpha(\alpha-1) \cdots(\alpha-k+1) ; \quad \alpha \in \mathbb{C}, k \in \mathbb{N} ;
$$

where $\alpha \underline{\underline{k}}$ is termed the " $k$-th falling (三 decreasing) factorial power of $\alpha$ ". Notice that we have defined $0^{\underline{0}}:=1$, hence a linear combination such as $\sum_{i=1}^{n} a_{i}\left(\alpha_{i}\right)^{\underline{0}}$ simply means $\sum_{i=1}^{n} a_{i}$ whatever the $\alpha_{i}$ 's.

- The symbol $W\left(u_{1}(x), \ldots, u_{i}(x)\right)$ denotes the Wronskian determinant of the ordered $i$-tuple $\left(u_{1}(x), \ldots, u_{i}(x)\right),(i-1)$ times differentiable at the specified point $x ; W(u(x)):=u(x)$.

- Whenever no ambiguity arises the notations:

$$
\operatorname{det}\left[c_{i, j}\right]_{i=1, \ldots, n}^{j=1, \ldots, n} ; \quad \operatorname{det}\left[c_{i, 1}, \ldots, c_{i, n}\right]_{i=1, \ldots, n} \equiv\left|c_{i, 1}, \ldots, c_{i, n}\right|_{i=1, \ldots, n}
$$

respectively denote the determinant of the matrix $\left(c_{i, j}\right)_{i=1, \ldots, n}^{j=1, \ldots, n}$ and the determinant of the matrix whose $i$-th row is $c_{i, 1}, \ldots, c_{i, n}$.

- Meaning of some asymptotic notations:

$$
\begin{gathered}
f(x) \preceq g(x), x \rightarrow x_{0}, \Longleftrightarrow g(x) \succeq f(x), x \rightarrow x_{0}, \stackrel{\text { def }}{\Longleftrightarrow} f(x)=O(g(x)), x \rightarrow x_{0} ; \\
f(x) \ll g(x), x \rightarrow x_{0}, \Longleftrightarrow g(x) \gg f(x), x \rightarrow x_{0}, \stackrel{\text { def }}{\Longleftrightarrow} f(x)=o(g(x)), x \rightarrow x_{0} ; \\
f(x)=\left\{\begin{array}{l}
+\infty(g(x)) \\
-\infty(g(x)), x \rightarrow x_{0}, \stackrel{\text { def }}{\Longleftrightarrow} f(x)=v(x) g(x) \quad \text { where } \quad \lim _{x \rightarrow x_{0}} v(x)=\left\{\begin{array}{l}
+\infty \\
-\infty
\end{array} ;\right.
\end{array}\right. \\
\qquad\left\{\begin{array}{l}
f(x) \asymp g(x), x \rightarrow x_{0}, \Longleftrightarrow g(x) \asymp f(x), x \rightarrow x_{0}, \\
\text { (to be read “ } \left.f \text { and } g \text { asymptotically similar as } x \rightarrow x_{0} ”\right)
\end{array}\right. \\
\Longleftrightarrow\left\{\begin{array}{l}
0<c_{1}|f(x)| \leq|g(x)| \leq c_{2}|f(x)| \text { on some deleted neighborhood of } x_{0}, \\
\text { with } c_{1}, c_{2} \text { positive constants. }
\end{array}\right.
\end{gathered}
$$


- Propositions are numbered consecutively irrespective of their labelling as lemma, theorem and so on.

Notations for iterated natural logarithms and exponentials:

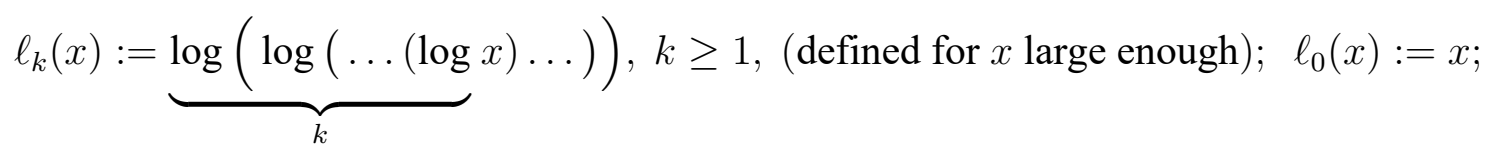

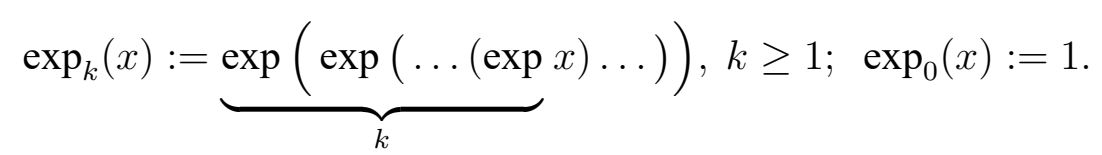

The following unified notation for each $k \in \mathbb{Z}$ may be convenient:

$$
\ell_{k}(x):= \begin{cases}\ell_{k}(x) & \text { if } k \geq 1 \\ x & \text { if } k=0 \\ \exp _{-k}(x) & \text { if } k \leq-1\end{cases}
$$

due to the operational formulas for inversion and composition:

$$
\left(\ell_{k}\right)^{-1}=\ell_{-k} ; \quad \ell_{h} \circ \ell_{k}=\ell_{h+k} \quad \forall h, k \in \mathbb{Z} .
$$

Their derivatives are:

$$
\frac{d}{d x} \ell_{k}(x)=\prod_{i=0}^{k-1} \frac{1}{\ell_{i}(x)}, k \geq 1 ; \quad \frac{d}{d x} \exp _{k}(x)=\prod_{i=0}^{k} \exp _{i}(x), k \geq 1 .
$$

The special definitions for $\ell_{0}$ and $\exp _{0}$ are agreements: that for $\ell_{0}$ makes (22) true $\forall h, k \in \mathbb{Z}$; that for $\exp _{0}$ is conveniently used in formulas involving logarithmic derivatives of iterated exponentials though some authors define $\exp _{0}(x):=x$.

Useful formulas for Wronskians.

For later references we explicitly mention two classic formulas concerning the Wronskians

$$
W\left(u_{1}(x), \ldots, u_{n}(x)\right):=\operatorname{det}\left[u_{1}^{(i)}(x), \ldots, u_{n}^{(i)}(x)\right]_{i=0, \ldots, n-1} .
$$

The Hesse-Christoffel's identity

$$
W\left(v(x) u_{1}(x), \ldots, v(x) u_{n}(x)\right)=(v(x))^{n} \cdot W\left(u_{1}(x), \ldots, u_{n}(x)\right),
$$

is valid under the required order of differentiability for $v, u_{i}$ regardless of the sign of $v$. The identity

$$
W\left(u_{1}(g(x)), \ldots, u_{n}(g(x))\right)=\left(g^{\prime}(x)\right)^{n(n-1) / 2} \cdot W\left(u_{1}(y), \ldots, u_{n}(y)\right),
$$

is valid after setting $y=g(x)$ in the right-hand side and under the required order of differentiability for $g, u_{i}$. The left-hand side in (26) involves derivatives with respect to the variable $x$ of the composite functions $u_{i}(g(x))$ whereas the right-hand side involves $g$ only as the factor $g^{\prime}$ and the derivatives in the Wronskian are those of the functions $u_{i}(y)$ with respect to $y$.

\section{A Brief Summary of the Concepts of Higher-Order Regular, Smooth and Rapid Variation}

The concepts to be defined refer to subclasses of functions strictly positive on some neighborhood of $+\infty$ and which are either regularly varying or rapidly varying at $+\infty$ in the "strong sense" that the following limit exists in the extended real line:

$$
\lim _{x \rightarrow+\infty} x f^{\prime}(x) / f(x) \equiv \alpha,-\infty \leq \alpha \leq+\infty,\left\{\begin{array}{l}
\text { regular variation if } \alpha \in \mathbb{R} \\
\text { rapid variation if } \alpha= \pm \infty
\end{array}\right.
$$


Here "strong sense" refers to the fact that we are considering: (i) $C^{1}$-functions or, at least, absolutely continuous functions; (ii) the definitions involving the logarithmic derivative rather than the weaker definitions based on suitable asymptotic functional equations for measurable functions. A possible notation for the corresponding class is $\mathcal{R}_{\alpha}(+\infty)$ and $\alpha$ is the "index of variation". If we take account of similar behaviors for some of the higher derivatives we are led to study three (not two!) classes of functions according to the asymptotic behavior of the ratios " $f^{(k)}(x) / f(x)$ ". And here are the main concepts about these classes of functions introduced and extensively discussed in [6;7].

Definition 1. (Higher-Order Regular Variation). A function $f \in A C^{n-1}[T,+\infty), n \geq 1$, is termed "regularly varying at $+\infty$ (in the strong sense) of order $n$ " if each of the functions $|f|,\left|f^{\prime}\right|, \ldots,\left|f^{(n-1)}\right|$ never vanishes on a neighborhood of $+\infty$ and is regularly varying at $+\infty$ with its own index of variation. If this is the case we use the notation:

$$
f \in\left\{\mathcal{R}_{\alpha}(+\infty) \text { of order } n\right\}, \quad \alpha:=\text { " the index of regular variation of } f \text { " } \in \mathbb{R} .
$$

Whenever needed we denote the indexes of the derivatives as follows:

$$
\left|f^{(k)}\right| \in \mathcal{R}_{\alpha_{k}}(+\infty), 0 \leq k \leq n-1 ; \alpha_{0} \equiv \alpha .
$$

The following relations hold true between the indexes $\alpha_{k}$ :

$$
\begin{gathered}
\alpha \notin\{0,1, \ldots, n-2\}, n \geq 2 \Rightarrow \alpha_{k}=\alpha-k, 1 \leq k \leq n-1 ; \\
\alpha \equiv k_{0} \in\{0,1, \ldots, n-2\}, n \geq 2 \Rightarrow \begin{cases}\alpha_{k}=k_{0}-k, & 0 \leq k \leq k_{0} \\
\alpha_{k_{0}+1}=\beta, & \text { for some } \beta \leq-1 ; \\
\alpha_{k}=\beta-\left(k-k_{0}+1\right), & k_{0}+1 \leq k \leq n-1 .\end{cases}
\end{gathered}
$$

If $f \in\left\{\mathcal{R}_{0}(+\infty)\right.$ of order $\left.n\right\}$, with $n \geq 2$, we have

$$
\alpha_{k+1}<\alpha_{k}<\alpha_{1} \leq-1, \quad(2 \leq k \leq n-1),
$$

hence it would be misleading to say that " $f$ is slowly varying at $+\infty$ (in the strong sense) of order $n$ " as usual in the case $n=1$. Think of the functions " $f(x):=1+x^{\delta}, \delta<0$ ".

Proposition 1. (Principal Parts of Higher Derivatives).

(I) If $f \in\left\{\mathcal{R}_{\alpha}(+\infty)\right.$ of order $\left.n\right\}, n \geq 1$, then the relations

$$
f^{(k)}(x) / f(x)=\alpha(\alpha-1) \cdots(\alpha-k+1) x^{-k}+o\left(x^{-k}\right) \equiv \alpha^{\underline{k}} x^{-k}+o\left(x^{-k}\right), x \rightarrow+\infty, 1 \leq k \leq n,
$$

hold true whichever $\alpha \in \mathbb{R}$ may be. For $\alpha \neq 0$ they may be written as

$$
f^{(k)}(x)=\frac{(\alpha-1) \cdots(\alpha-k+1)}{\alpha^{k-1}} \frac{\left(f^{\prime}(x)\right)^{k}}{(f(x))^{k-1}}+o\left(\frac{\left(f^{\prime}(x)\right)^{k}}{(f(x))^{k-1}}\right), x \rightarrow+\infty, 1 \leq k \leq n,
$$

or, equivalently, in the form used in (11).

(II) (Partial Converse). If $f \in A C^{n-1}[T,+\infty), n \geq 2$, then $f \in\left\{\mathcal{R}_{\alpha}(+\infty)\right.$ of order $\left.n\right\}$ for some real number $\alpha \notin\{0,1, \ldots, n-2\}$ iff the following relations hold true:

$$
f^{(k)}(x) / f(x)=\gamma_{k} x^{-k}+o\left(x^{-k}\right), x \rightarrow+\infty, 1 \leq k \leq n,
$$

with suitable constants $\gamma_{k}$ such that

$$
\left.\gamma_{1}, \ldots, \gamma_{n-1} \neq 0 ; \quad \text { (no restriction on } \gamma_{n}\right) \text {. }
$$

If this is the case then: $\gamma_{k}=\alpha^{\underline{k}}, 1 \leq k \leq n$. 
Relations (35) are very useful for applications and to avoid any restrictions on the $\gamma_{k}$ 's the following class is introduced.

Definition 2. (Higher-Order Smooth Variation). A function $f \in A C^{n-1}[T,+\infty), n \geq 1, f(x) \neq$ $0 \forall x$ large enough, is termed "smoothly varying at $+\infty$ of order $n$ and index $\alpha$ " if the four equivalent properties in the following proposition, referred to $|f|$, are satisfied. We denote this class by: $\left\{S \mathcal{R}_{\alpha}(+\infty)\right.$ of order $\left.n\right\}$.

Proposition 2. (Several Characterizations). For an $f \in A C^{n-1}[T,+\infty), n \geq 1, f(x)>0 \forall x$ large enough, the following four sets of asymptotic relations, for a fixed $\alpha \in \mathbb{R}$, are equivalent to each other:

$$
\begin{aligned}
& x^{k} f^{(k)}(x) / f(x)=\alpha(\alpha-1) \cdots(\alpha-k+1)+o(1), x \rightarrow+\infty, 1 \leq k \leq n ; \\
& \left\{\begin{array}{l}
x f^{\prime}(x) / f(x)=\alpha+o(1), x \rightarrow+\infty ; \\
\left(x f^{\prime}(x) / f(x)\right)^{(k)}=o\left(x^{-k}\right), x \rightarrow+\infty, 1 \leq k \leq n ;
\end{array}\right. \\
& \left\{\begin{array}{l}
f^{\prime}(x) / f(x)=\alpha x^{-1}+o\left(x^{-1}\right), x \rightarrow+\infty ; \\
\left(f^{\prime}(x) / f(x)\right)^{(k)}=\alpha \cdot D^{k} x^{-1}+o\left(x^{-k-1}\right), x \rightarrow+\infty, 1 \leq k \leq n ;
\end{array}\right. \\
& \left\{\begin{array}{l}
\phi(x):=\log \left[f\left(e^{x}\right)\right] ; \phi^{\prime}(x)=\alpha+o(1), x \rightarrow+\infty ; \\
\phi^{(k)}(x)=o(1), x \rightarrow+\infty, 2 \leq k \leq n .
\end{array}\right.
\end{aligned}
$$

For $\alpha \neq 0$ and $n \geq 2$ the further pair of conditions may be added:

$$
\left\{\begin{array}{l}
x f^{\prime}(x) / f(x) \sim \alpha, x \rightarrow+\infty,(\alpha \neq 0) \\
x^{k} f^{(k)}(x) / f(x)=\gamma_{k}+o(1), 2 \leq k \leq n, x \rightarrow+\infty
\end{array}\right.
$$

for some unspecified constants $\gamma_{k}$. The following inclusions hold true:

$\left\{\mathcal{R}_{\alpha}(+\infty)\right.$ of order $\left.n\right\}=\left\{S \mathcal{R}_{\alpha}(+\infty)\right.$ of order $\left.n\right\}$ if $n=1$ or $\{n \geq 2, \alpha \neq 0,1, \ldots, n-2\}$; $\left\{\mathcal{R}_{\alpha}(+\infty)\right.$ of order $\left.n\right\} \underset{f}{\subset}\left\{S \mathcal{R}_{\alpha}(+\infty)\right.$ of order $\left.n\right\}$ otherwise;

the reason of the strict inclusion being that some derivatives of a smoothly-varying function may vanish or change sign infinitely often.

Definition 3. (Higher-Order Rapid Variation).

(I) (First order). A function $f \in A C^{1}[T,+\infty)$, is called "rapidly varying at $+\infty$ (of order 1 ) in the strong restricted sense" if

$$
\begin{cases}f(x), f^{\prime}(x) \neq 0 & \forall x \text { large enough; } \\ f(x) / f^{\prime}(x)=o(x), & x \rightarrow+\infty \\ \left(f(x) / f^{\prime}(x)\right)^{\prime}=o(1), & x \rightarrow+\infty\end{cases}
$$

(II) (Higher order). A function $f \in A C^{n}[T,+\infty)$, is called "rapidly varying at $+\infty$ of order $n \geq 2$ in the strong restricted sense" if all the functions $f, f^{\prime}, \ldots, f^{(n-1)}$ are rapidly varying at $+\infty$ in the strong restricted sense and this amounts to say that the following conditions hold true as $x \rightarrow+\infty$ :

$$
\begin{gathered}
f^{(k)}(x) \neq 0 \forall x \text { large enough and } 0 \leq k \leq n ; \\
f(x) / f^{\prime}(x)=o(x) ; f^{\prime}(x) / f^{\prime \prime}(x)=o(x) ; \ldots ; f^{(n-1)}(x) / f^{(n)}(x)=o(x) ; \\
\left(f(x) / f^{\prime}(x)\right)^{\prime}=o(1) ;\left(f^{\prime}(x) / f^{\prime \prime}(x)\right)^{\prime}=o(1) ; \ldots ;\left(f^{(n-1)}(x) / f^{(n)}(x)\right)^{\prime}=o(1) .
\end{gathered}
$$


If $f$ is rapidly varying at $+\infty$ of order $n \geq 2$ in the previous strong restricted sense then it can be proved that all the functions $f, f^{\prime}, \ldots, f^{(n-1)}$ belong to the same class, either $\mathcal{R}_{-\infty}(+\infty)$ or $\mathcal{R}_{+\infty}(+\infty)$, hence we shall use notation $f \in\left\{\mathcal{R}_{ \pm \infty}(+\infty)\right.$ of order $\left.n\right\}$ to denote that $f$ enjoys the properties in (44)-(45)-(46) plus the corresponding value " $\pm \infty$ " of the limit in (27). The set of conditions in (46) is added in view of meaningful applications. In most cases we shall not be interested in functions satisfying (44)-(45) but not (46), and so we use no additional notation to highlight the "strong restricted sense" where "strong" refers to the existence of the limit in (27) and "restricted" to the conditions in (46).

Proposition 3. (Principal Parts of Higher Derivatives). Let $f \in A C^{n}[T,+\infty)$ and conditions in (44) be satisfied. Then $f \in\left\{\mathcal{R}_{+\infty}(+\infty)\right.$ of order $\left.n\right\} \cup\left\{\mathcal{R}_{-\infty}(+\infty)\right.$ of order $\left.n\right\}$, i.e. conditions in (46) hold true, if and only if the following four equivalent sets of conditions are satisfied:

$$
\begin{gathered}
\left\{\begin{array}{l}
f^{\prime}(x) / f(x) \sim f^{\prime \prime}(x) / f^{\prime}(x) \sim \cdots \sim f^{(n)}(x) / f^{(n-1)}(x) \sim f^{(n+1)}(x) / f^{(n)}(x) \\
\text { i.e. } D_{\ell}\left(f^{(k)}(x)\right) \sim D_{\ell}(f(x)), x \rightarrow+\infty, 1 \leq k \leq n
\end{array}\right. \\
f^{(k+2)}(x) \sim\left(f^{(k+1)}(x)\right)^{2} / f^{(k)}(x), x \rightarrow+\infty, 0 \leq k \leq n-1 ; \\
f^{(k+2)}(x) / f(x) \sim\left(f^{\prime}(x) /(f(x))^{k+2} \equiv\left(D_{\ell}(f(x))\right)^{k+2}, x \rightarrow+\infty, 0 \leq k \leq n-1\right.
\end{gathered}
$$

It follows that even $f^{(n+1)}(x) \neq 0$ for almost all $x$ large enough. Relations in (49) are formally obtained from those in (34) as the index $\alpha$ tends to $+\infty$. A different way of writing relations in (45) yields the asymptotic scale:

$$
f^{(n)}(x) \gg x^{-1} f^{(n-1)}(x) \gg x^{-2} f^{(n-2)}(x) \gg \cdots \gg x^{-(n-1)} f^{\prime}(x) \gg x^{-n} f(x), x \rightarrow+\infty .
$$

- Typical functions in the class $\left\{\mathcal{R}_{\alpha}(+\infty)\right.$ of any order $\left.n \in \mathbb{N}\right\}, \alpha \in \mathbb{R}$, are:

$$
\left\{\begin{array}{l}
x^{\alpha} \cdot\left[\prod_{k=1}^{p_{1}}\left(\ell_{k}(x)\right)^{\beta_{k}}\right] \cdot \exp \left[\sum_{k=1}^{p_{2}} c_{k}(\log x)^{\gamma_{k}}\right] \cdot \exp \left[\sum_{k=2}^{p_{3}} d_{k}\left(\ell_{k}(x)\right)^{\delta_{k}}\right], \\
\alpha, \beta_{k}, c_{k}, d_{k} \in \mathbb{R} ; 0<\gamma_{k}<1 ; 0<\delta_{k} .
\end{array}\right.
$$

- Examples of functions which are smoothly varying but not regularly varying:

$$
\begin{cases}f(x):=\text { constant } \neq 0 & \in\left\{S \mathcal{R}_{0}(+\infty) \text { of order } n\right\} \backslash\left\{\mathcal{R}_{0}(+\infty) \text { of order } n\right\} \text { for each } n \geq 2 ; \\
f(x):=1+x^{-\delta}, \delta>0, & \in\left\{\mathcal{R}_{0}(+\infty) \text { of any order } n \geq 2\right\} ; \\
f(x):=1+e^{-x} & \in\left\{S \mathcal{R}_{0}(+\infty) \text { of order } n\right\} \backslash\left\{\mathcal{R}_{0}(+\infty) \text { of order } n\right\} \text { for each } n \geq 2 ; \\
f(x):=\left\{\begin{array}{l}
2+\sin \left[(\log x)^{\delta}\right] \\
\text { with } 0<\delta<1
\end{array}\right. & \in\left\{S \mathcal{R}_{0}(+\infty) \text { of order } n\right\} \backslash\left\{\mathcal{R}_{0}(+\infty) \text { of order } n\right\} \text { for each } n \geq 2 .\end{cases}
$$

- Typical functions in the class $\left\{\mathcal{R}_{-\infty}(+\infty)\right.$ of any order $\left.n \in \mathbb{N}\right\}$, are:

$$
\left\{\begin{array}{l}
R_{\alpha}(x) \cdot \exp \left(c_{1} x^{\gamma_{1}}+\cdots+c_{k} x^{\gamma_{k}}\right),\left(c_{i}<0, \gamma_{i}>0 \forall i\right), \\
R_{\alpha} \in\left\{\mathcal{R}_{\alpha}(+\infty) \text { of any order } n \in \mathbb{N}\right\} .
\end{array}\right.
$$

- Typical functions in the class $\left\{\mathcal{R}_{+\infty}(+\infty)\right.$ of any order $\left.n \in \mathbb{N}\right\}$, are:

$$
\left\{\begin{array}{l}
R_{\alpha}(x) \cdot \exp \left(d_{1} x^{\delta_{1}}+\cdots+d_{k} x^{\delta_{k}}\right),\left(d_{i}, \delta_{i}>0 \forall i\right), \\
R_{\alpha} \in\left\{\mathcal{R}_{\alpha}(+\infty) \text { of any order } n \in \mathbb{N}\right\}
\end{array}\right.
$$


If $\phi$ is any functions of type (54) then $E(x):=\exp (c \phi(x)) \in\left\{\mathcal{R}_{0}(+\infty)\right.$ of any order $\left.\mathrm{n}\right\}$ for any constant $c \neq 0$ noticing that $E(x) \sim 1, x \rightarrow+\infty$. On the contrary, if $\phi$ is any functions of type (55) then:

$$
\exp (c \phi(x)) \in\left\{\begin{array}{l}
\left\{\mathcal{R}_{-\infty}(+\infty) \text { of any order } n\right\} \text { if } c<0 \\
\left\{\mathcal{R}_{+\infty}(+\infty) \text { of any order } n\right\} \text { if } c>0
\end{array}\right.
$$

\section{General Results on the Asymptotic Behaviors of Vandermonde-like Determinants}

In this section we obtain some basic results on the asymptotic behavior of a determinant of the form

$$
V\left(g_{1}(x), \ldots, g_{n}(x)\right):=\left|\begin{array}{cccc}
1 & 1 & \ldots & 1 \\
g_{1}(x) & g_{2}(x) & \ldots & g_{n}(x) \\
\vdots & \vdots & & \vdots \\
\left(g_{1}(x)\right)^{n-1} & \left(g_{2}(x)\right)^{n-1} & \ldots & \left(g_{n}(x)\right)^{n-1}
\end{array}\right|
$$

whose elements $g_{i}$ are aymptotically equivalent to simpler functions $f_{i}$. For later references it is necessary to state a few elementary well-known results and techniques about Vandermonde determinants (三 Vandermondians).

Lemma 4. (General properties of Vandermondians).

1) If $\left(c_{1}, \ldots, c_{n}\right)$ is an ordered $n$-tuple, $n \geq 2$, of complex numbers, its Vandermondian is defined as the determinant:

$$
V\left(c_{1}, \ldots, c_{n}\right):=\left|\begin{array}{cccc}
1 & 1 & \ldots & 1 \\
c_{1} & c_{2} & \ldots & c_{n} \\
c_{1}^{2} & c_{2}^{2} & \ldots & c_{n}^{2} \\
\vdots & \vdots & & \vdots \\
c_{1}^{n-1} & c_{2}^{n-1} & \ldots & c_{n}^{n-1}
\end{array}\right| \equiv \operatorname{det}\left[\left(c_{1}\right)^{i}, \ldots,\left(c_{n}\right)^{i}\right]_{i=0, \ldots, n-1}=\prod_{1 \leq i<j \leq n}\left(c_{j}-c_{i}\right) .
$$

Hence $V\left(c_{1}, \ldots, c_{n}\right) \neq 0$ iff $c_{1}, \ldots, c_{n}$ are distinct numbers. It is convenient to define $V\left(c_{1}\right):=1$.

2) The product in (58) explicitly reads

$$
\left(c_{2}-c_{1}\right)\left[\left(c_{3}-c_{2}\right)\left(c_{3}-c_{1}\right)\right]\left[\left(c_{4}-c_{3}\right)\left(c_{4}-c_{2}\right)\left(c_{4}-c_{1}\right)\right] \cdots\left[\left(c_{n}-c_{n-1}\right)\left(c_{n}-c_{n-2}\right) \cdots\left(c_{n}-c_{1}\right)\right],
$$

hence it contains " $1+2+\cdots+(n-1)=n(n-1) / 2$ " factors.

3) If the $c_{i}$ 's are such that $c_{i+1}-c_{i}=k \forall i$ then (58)-(59) imply:

$$
V(c, c+k, c+2 k, \ldots, c+(n-1) k)=1 ! 2 ! \ldots(n-1) ! k^{n(n-1) / 2}, n \geq 2,
$$

and in particular:

$$
V(c, c+1, c+2, \ldots, c+n-1)=\prod_{i=2}^{n-1} i !, n \geq 2 .
$$

4) The following simplification and reduction formulas hold true:

$$
\begin{gathered}
V\left(c c_{1}, \ldots, c c_{n}\right)=c^{n(n-1) / 2} \cdot V\left(c_{1}, \ldots, c_{n}\right), n \geq 2 ; \\
V\left(c_{1}+c, c_{2}+c, \ldots, c_{n}+c\right)=V\left(c_{1}, \ldots, c_{n}\right), n \geq 2 ; \\
\Rightarrow V\left(c_{1}, \ldots, c_{n}\right)=(-1)^{i-1}\left(\prod_{j=1, \ldots, n}^{j \neq i} c_{j}\right) \cdot V\left(c_{1}, \ldots, c_{i-1}, c_{i+1}, \ldots, c_{n}\right), n \geq 2 ;
\end{gathered}
$$




$$
V\left(c_{1}, \ldots, c_{n}\right)=V\left(0, c_{2}-c_{1}, \ldots, c_{n}-c_{1}\right)=\left(\prod_{i=2}^{n}\left(c_{i}-c_{1}\right)\right) \cdot V\left(c_{2}-c_{1}, \ldots, c_{n}-c_{1}\right), n \geq 2 .
$$

5) With the notations in (13) the identity holds true:

$$
\operatorname{det}\left[\left(c_{1}\right)^{j}, \ldots,\left(c_{n}\right)^{\frac{j}{j}}\right]_{j=0, \ldots, n-1} \equiv\left|\begin{array}{ccc}
1 & \ldots & 1 \\
c_{1} & \ldots & c_{n} \\
\left(c_{1}\right)^{2} & \ldots & \left(c_{n}\right)^{\underline{2}} \\
\ldots & \ldots & \ldots \\
\left(c_{1}\right)^{\frac{n-1}{2}} & \ldots & \left(c_{n}\right)^{\underline{n-1}}
\end{array}\right|=V\left(c_{1}, \ldots, c_{n}\right) .
$$

Proof. All formulas (60) to (65) are trivial consequences of the classical formula (58). We outline an elementary standard proof of (66) as we shall need its procedure in the sequel. We start from the determinant on the left in (66) and, for $n \geq 3$, subtract the second row from the third row so that the determinant is seen to equal

$$
\left|\begin{array}{ccc}
1 & \cdots & 1 \\
c_{1} & \cdots & c_{n} \\
\left(c_{1}\right)^{2} & \cdots & \left(c_{n}\right)^{2} \\
\left(c_{1}\right)^{\underline{3}} & \cdots & \left(c_{n}\right)^{\underline{3}} \\
\cdots & \cdots & \cdots \\
\left(c_{1}\right)^{\frac{n-1}{}} & \cdots & \left(c_{n}\right)^{\frac{n-1}{n}}
\end{array}\right|
$$

then from the fourth row we subtract a suitable linear combination of the preceding rows and so on. Step after step all the factorial powers change into standard powers and (66) follows.

An application of the identities (25)-(26) yields a simple proof for the explicit expression of the Wronskian of powers.

Lemma 5. (Wronskian of powers of a given function). If $g$ is $(n-1)$-times differentiable on an interval $J, n \geq 2$, and if $\alpha_{1}, \ldots, \alpha_{n}$ are any real distinct numbers then:

$$
W\left((g(x))^{\alpha_{1}}, \ldots,(g(x))^{\alpha_{n}}\right)=V\left(\alpha_{1}, \ldots, \alpha_{n}\right) \cdot(g(x))^{\alpha_{1}+\cdots+\alpha_{n}-[n(n-1) / 2]} \cdot\left(g^{\prime}(x)\right)^{n(n-1) / 2},
$$

for $x \in J$; and if $g$ is n-times differentiable then:

$$
\begin{aligned}
W\left(1,(g(x))^{\alpha_{1}}, \ldots,(g(x))^{\alpha_{n}}\right) & =\left(\prod_{i=1}^{n} \alpha_{i}\right) \cdot V\left(\alpha_{1}, \ldots, \alpha_{n}\right) \cdot(g(x))^{\alpha_{1}+\cdots+\alpha_{n}-[(n+1) n / 2]} \times \\
& \times\left(g^{\prime}(x)\right)^{(n+1) n / 2}, x \in J, n \geq 1 .
\end{aligned}
$$

Restrictions (if any) on the sign of $g$ are those imposed by the exponents $\alpha_{i}$. For the special choice $\alpha_{i}=i$ we get the classical formulas ascribed to Wronski:

$$
\begin{gathered}
W\left(g(x),(g(x))^{2}, \ldots,(g(x))^{n}\right)=\left(\prod_{i=1}^{n-1} i !\right)(g(x))^{n}\left(g^{\prime}(x)\right)^{n(n-1) / 2}, x \in J ; n \geq 2 ; \\
W\left(1, g(x),(g(x))^{2}, \ldots,(g(x))^{n}\right)=\left(\prod_{i=1}^{n} i !\right)\left(g^{\prime}(x)\right)^{(n+1) n / 2}, x \in J ; n \geq 1 .
\end{gathered}
$$

Proof. By (26) it is enough to prove (68) for $g(x) \equiv x$. This is trivial for $n=2$ and if, by induction, we assume it to be true for any $n$ numbers $\alpha_{1}, \ldots, \alpha_{n}$, then for $(n+1)$ numbers $\alpha_{1}, \ldots, \alpha_{n+1}$ we have:

$$
\begin{aligned}
& W\left(x^{\alpha_{1}}, \ldots, x^{\alpha_{n+1}}\right) \stackrel{(25)}{=} x^{(n+1) \alpha_{1}} \cdot W\left(1, x^{\alpha_{2}-\alpha_{1}}, \ldots, x^{\alpha_{n+1}-\alpha_{1}}\right) \\
= & \left(\prod_{i=2}^{n+1}\left(\alpha_{i}-\alpha_{1}\right)\right) \cdot V\left(\alpha_{2}-\alpha_{1}, \ldots, \alpha_{n+1}-\alpha_{1}\right) \cdot x^{(n+1) \alpha_{1}+\left[\sum_{i=2}^{n+1}\left(\alpha_{i}-\alpha_{1}\right)\right]-[(n+1) n / 2]} \\
\stackrel{(65)}{=} & V\left(\alpha_{1}, \alpha_{2}, \ldots, \alpha_{n+1}\right) \cdot x^{\alpha_{1}+\cdots+\alpha_{n+1}-[(n+1) n / 2]},
\end{aligned}
$$


which is (68) with $n$ replaced by $n+1$.

And here is the main result in this section concerning the behavior of a determinant of type $V\left(f_{1}(x)+o\left(f_{1}(x)\right), \ldots, f_{n}(x)+o\left(f_{n}(x)\right)\right.$.

Theorem 6. Let $g_{1}, \ldots, g_{n}$ and $f_{1}, \ldots, f_{n}, n \geq 2$, be functions defined on a deleted neighborhood of $x_{0} \in \overline{\mathbb{R}}$ and such that:

$$
g_{i}(x)=f_{i}(x)+o\left(f_{i}(x)\right), x \rightarrow x_{0}, 1 \leq i \leq n .
$$

Then: (I) The Vandermondians of the $g_{i}$ 's and the $f_{i}$ 's are linked by the asymptotic relation

$$
\begin{aligned}
& V\left(g_{1}(x), \ldots, g_{n}(x)\right) \equiv V\left(f_{1}(x)+o\left(f_{1}(x)\right), \ldots, f_{n}(x)+o\left(f_{n}(x)\right)\right)= \\
& =V\left(f_{1}(x), \ldots, f_{n}(x)\right)+o\left(\sum_{\left(p_{1}, \ldots, p_{n}\right) \in \mathcal{P}} \prod_{i=1}^{n}\left|f_{i}(x)\right|^{p_{i}}\right), x \rightarrow x_{0},
\end{aligned}
$$

where $\mathcal{P}$ denotes the set of all permutations of $(0,1, \ldots, n-1)$. The "o"-term in $(74)$ is a true remainder under some conditions on the $f_{i}$ 's which we are going to make explicit.

(II) Assume further the following relationships between any pair of consecutive $f_{i}$ s:

either $f_{i}(x) \gg f_{i+1}(x), x \rightarrow x_{0}, \quad$ or $\quad f_{i}(x) \asymp f_{i+1}(x), x \rightarrow x_{0}$, for each fixed $i$,

the last symbol being defined in (18). Then (74) takes the simpler form:

$$
\begin{aligned}
& V\left(f_{1}(x)+o\left(f_{1}(x)\right), \ldots, f_{n}(x)+o\left(f_{n}(x)\right)\right)= \\
= & V\left(f_{1}(x), \ldots, f_{n}(x)\right)+o\left(\prod_{i=1}^{n-1}\left|f_{i}(x)\right|^{n-i}\right), x \rightarrow x_{0}
\end{aligned}
$$

and

$$
V\left(f_{1}(x), \ldots, f_{n}(x)\right)=O\left(\prod_{i=1}^{n-1}\left|f_{i}(x)\right|^{n-i}\right), x \rightarrow x_{0} ;
$$

noticing the lack of $f_{n}$ which is one of the functions $f_{i}$ with the lowest growth-order.

Formula (76) is the basic formula in our theory; in any specific case one has to make explicit the principal part of each factor $f_{j}(x)-f_{i}(x)$ in the factorized expression of $V\left(f_{1}(x), \ldots, f_{n}(x)\right)$. Parts (III) and (IV) of this theorem give the formulas for the two most meaningful cases.

(III) If $\left(f_{1}, \ldots, f_{n}\right)$ is an asymptotic scale, i.e.

$$
f_{1}(x) \gg \cdots \gg f_{n}(x), x \rightarrow x_{0},
$$

then (76) becomes:

$$
\begin{aligned}
& V\left(f_{1}(x)+o\left(f_{1}(x)\right), \ldots, f_{n}(x)+o\left(f_{n}(x)\right)\right)= \\
= & (-1)^{n(n-1) / 2}\left(\prod_{i=1}^{n-1}\left(f_{i}(x)\right)^{n-i}\right)+o\left(\prod_{i=1}^{n-1}\left|f_{i}(x)\right|^{n-i}\right), x \rightarrow x_{0} .
\end{aligned}
$$

(IV) If all the $f_{i}$ 's have the same growth-order in the sense that:

$$
f_{i}(x)=c_{i} f(x)+o(f(x)), x \rightarrow x_{0}, 1 \leq i \leq n,
$$


for a fixed $f$ and arbitrary constants $c_{i}$ then:

$$
\begin{aligned}
& V\left(c_{1} f(x)+o(f(x)), \ldots, c_{n} f(x)+o(f(x))\right)= \\
= & V\left(c_{1}, \ldots, c_{n}\right) \cdot(f(x))^{n(n-1) / 2}+o\left(|f(x)|^{n(n-1) / 2}\right), x \rightarrow x_{0} .
\end{aligned}
$$

In the special case $f(x) \equiv 1$ we get:

$$
V\left(c_{1}+o(1), \ldots, c_{n}+o(1)\right) \equiv\left|\begin{array}{ccc}
1 & \ldots & 1 \\
c_{1}+o(1) & \ldots & c_{n}+o(1) \\
\vdots & & \vdots \\
c_{1}^{n-1}+o(1) & \ldots & c_{n}^{n-1}+o(1)
\end{array}\right|=V\left(c_{1}, \ldots, c_{n}\right)+o(1) .
$$

Formulas (81)-(82) hold true even if some of the constants $c_{i}$ 's (which may possibly equal zero) coincide; obviously the formulas specify the exact asymptotic behaviors of the Vandermondians on the left only when $V\left(c_{1}, \ldots, c_{n}\right) \neq 0$ including the case that $c_{i}=0$ for one value of $i$.

In the special case that all the $c_{i}$ 's in (80) coincide and two-term asymptotic expansions for the $f_{i}$ 's are known, namely:

$$
f_{i}(x)=f(x)+\bar{c}_{i} h_{i}(x)+o\left(h_{i}(x)\right), x \rightarrow x_{0},
$$

for a fixed $f$ and arbitrary constants $\bar{c}_{i}$ then, by the simplification formula (63), we get:

$$
V\left(f_{1}(x), \ldots, f_{n}(x)\right)=V\left(\bar{c}_{1} h_{1}(x)+o\left(h_{1}(x)\right), \ldots, \bar{c}_{n} h_{n}(x)+o\left(h_{n}(x)\right)\right), x \rightarrow x_{0},
$$

and then try to apply one of the foregoing results.

(V) Suppose that the functions $f_{i}$ may be grouped in several ordered $i_{k}$-tuples

$$
\left(f_{1}, \ldots, f_{i_{1}}\right),\left(f_{i_{1}+1}, \ldots, f_{i_{2}}\right), \ldots,\left(f_{i_{k-1}+1}, \ldots, f_{i_{k}}\right),
$$

each of which either forms an asymptotic scale at $x_{0}$ or contains elements with the same growthorder in the sense of (80); moreover the functions in each group have smaller growth-orders than the functions in the preceding group, i.e.

$$
f_{i_{1}} \gg f_{i_{1}+1} ; \quad f_{i_{2}} \gg f_{i_{2}+1} ; \ldots ; f_{i_{k-1}} \gg f_{i_{k-1}+1}, x \rightarrow x_{0} .
$$

This implies that the grouping may always be done so that two consecutive groups are of different types. Then an asymptotic formula like (79) holds true wherein the constant factor $(-1)^{n(n-1) / 2}$ is replaced by a suitable constant solely dependent on the $c_{i}$ 's. Such a constant is zero iff two of the $c_{i}$ 's coincide in at least one of the groups formed by elements with the same growth-order: the same circumstance as in (81). Such a general statement follows from thoroughly examining the case of two groups. In fact let $n, m \in \mathbb{N}$ be such that:

$$
\left\{\begin{array}{l}
n \geq 3,1 \leq m \leq n-2 ; f_{1}(x) \gg \cdots \gg f_{m}(x), x \rightarrow x_{0} \\
f_{i}(x)=c_{i} f(x)+o(f(x)), x \rightarrow x_{0}, \quad m+1 \leq i \leq n
\end{array}\right.
$$

and

$$
\text { either } \quad f_{1} \gg \cdots \gg f_{m} \gg f, \quad \text { or } \quad f \gg f_{1} \gg \cdots \gg f_{m}, x \rightarrow x_{0} .
$$

If " $f_{1} \gg \cdots \gg f_{m} \gg f$ " then:

$$
\begin{aligned}
& V\left(f_{1}(x)+o\left(f_{1}(x)\right), \ldots, f_{n}(x)+o\left(f_{n}(x)\right)\right)= \\
= & (-1)^{n(n-1) / 2} \cdot V\left(c_{n}, c_{n-1}, \ldots, c_{m+1}\right) \cdot(f(x))^{(n-m)(n-m-1) / 2} \cdot\left(\prod_{i=1}^{m}\left(f_{i}(x)\right)^{n-i}\right)+ \\
& +o\left(|f(x)|^{(n-m)(n-m-1) / 2} \prod_{i=1}^{m}\left|f_{i}(x)\right|^{n-i}\right), x \rightarrow x_{0},
\end{aligned}
$$


noticing the reverse order of the $c_{i}$ 's in $V\left(c_{n}, c_{n-1}, \ldots, c_{m+1}\right)$. If " $f \gg f_{1} \gg \cdots \gg f_{m}$ " then:

$$
\begin{aligned}
& V\left(f_{1}(x)+o\left(f_{1}(x)\right), \ldots, f_{n}(x)+o\left(f_{n}(x)\right)\right)= \\
= & (-1)^{[n(n-1) / 2]+m(n-m)} \cdot\left(\prod_{i=m+1}^{n} c_{i}\right)^{m} \cdot V\left(c_{n}, c_{n-1}, \ldots, c_{m+1}\right) \times \\
& \times(f(x))^{(n-m)(m+n-1) / 2} \cdot\left(\prod_{i=1}^{m-1}\left(f_{i}(x)\right)^{m-i}\right)+ \\
& +o\left(|f(x)|^{(n-m)(m+n-1) / 2} \prod_{i=1}^{m-1}\left|f_{i}(x)\right|^{m-i}\right), x \rightarrow x_{0},
\end{aligned}
$$

For the special case $f(x) \equiv 1$ and $c_{m+1}>c_{m+2}>\cdots>c_{n}$, the formulas are:

$$
\begin{aligned}
& V\left(f_{1}(x)+o\left(f_{1}(x)\right), \ldots, f_{m}(x)+o\left(f_{m}(x)\right), c_{m+1}+o(1), \ldots, c_{n}+o(1)\right) \sim \\
& \sim(-1)^{n(n-1) / 2} \cdot V\left(c_{n}, c_{n-1}, \ldots, c_{m+1}\right) \cdot\left(\prod_{i=1}^{m}\left(f_{i}(x)\right)^{n-i}\right), x \rightarrow x_{0}, \text { if } f_{1} \gg \cdots \gg f_{m} \gg 1 \\
& V\left(f_{1}(x)+o\left(f_{1}(x)\right), \ldots, f_{m}(x)+o\left(f_{m}(x)\right), c_{m+1}+o(1), \ldots, c_{n}+o(1)\right) \sim \\
& \sim(-1)^{[n(n-1) / 2]+m(n-m)} \cdot\left(\prod_{i=m+1}^{n} c_{i}\right)^{m} \cdot V\left(c_{n}, c_{n-1}, \ldots, c_{m+1}\right) \times \\
& \quad\left(\prod_{i=1}^{m-1}\left(f_{i}(x)\right)^{m-i}\right), x \rightarrow x_{0}, \text { if } 1 \gg f_{1} \gg \cdots \gg f_{m} .
\end{aligned}
$$

Proof. (I) As $\left[f_{i}(x)+o\left(f_{i}(x)\right)\right]^{k}=\left(f_{i}(x)\right)^{k}+o\left(\left(f_{i}(x)\right)^{k}\right)$, the determinant in (57) has the following form:

$$
\left|\begin{array}{ccc}
1 & \ldots & 1 \\
f_{1}(x)+o\left(f_{1}(x)\right) & \ldots & f_{n}(x)+o\left(f_{n}(x)\right) \\
\vdots & & \vdots \\
\left(f_{1}(x)\right)^{n-1}+o\left(\left(f_{1}(x)\right)^{n-1}\right) & \ldots & \left(f_{n}(x)\right)^{n-1}+o\left(\left(f_{n}(x)\right)^{n-1}\right)
\end{array}\right|
$$

and each term of its development is a product of type

$$
\pm \prod_{i=1}^{n}\left(f_{i}(x)\right)^{p_{i}}+o\left(\prod_{i=1}^{n}\left|f_{i}(x)\right|^{p_{i}}\right)
$$

with the proper sign and where $\left(p_{1}, \ldots, p_{n}\right)$ is a permutation of $(0,1, \ldots, n-1)$, hence it equals the corresponding term in the development of the determinant $V\left(f_{1}(x), \ldots, f_{n}(x)\right)$ plus the specified remainder. In (94) the factor $\left(f_{i}(x)\right)^{0}$ stands for the number " 1 " appearing in the $i$-th column. Summing up all the terms we get (74). (II) Under conditions in (75) a moment's reflection shows that one of the terms with maximal growth-order among those appearing in the sum inside the " 0 "-term in (74) is

$$
\left|f_{1}(x)\right|^{n-1} \cdot\left|f_{2}(x)\right|^{n-2} \cdots\left|f_{n-1}(x)\right|^{1} \cdot\left|f_{n}(x)\right|^{0} .
$$

In fact if we interchange the exponents of two factors in (95) replacing, say, $\left|f_{i}\right|^{n-i} \cdot\left|f_{j}\right|^{n-j}$ with $\left|f_{i}\right|^{n-j} \cdot\left|f_{j}\right|^{n-i}, i<j$, and leave unchanged the other factors we would obtain a function with a non-higher growth-order as

$$
\left(\left|f_{i}\right|^{n-j} \cdot\left|f_{j}\right|^{n-i}\right) /\left(\left|f_{i}\right|^{n-i} \cdot\left|f_{j}\right|^{n-j}\right)=\left|f_{j} / f_{i}\right|^{j-i}\left\{\begin{array}{cl}
\text { either } & =o(1) \\
\text { or } & \asymp 1 .
\end{array}\right.
$$


Hence the " $O$ "-term in (74) takes the form in (76). Moreover $f_{j}(x)-f_{i}(x)=O\left(f_{i}(x)\right)$ for $j>i$ so that (77) follows from the explicit factorization in (58). (III) If (78) holds true then we start from

$$
V\left(f_{1}(x), \ldots, f_{n}(x)\right)=\prod_{1 \leq i<j \leq n}\left(f_{j}(x)-f_{i}(x)\right) .
$$

Taking account that $f_{j}(x)=o\left(f_{i}(x)\right)$ for $j>i$, and using the factorization in (58) we get:

$$
V\left(f_{1}(x), \ldots, f_{n}(x)\right)=(-1)^{n(n-1) / 2} f_{1}^{n-1} f_{2}^{n-2} \cdots f_{n-2}^{2} f_{n-1}+o\left(\prod_{i=1}^{n-1}\left|f_{i}(x)\right|^{n-i}\right)
$$

which is (79). (IV) Under condition (80) we have $f_{j}(x)-f_{i}(x)=\left(c_{j}-c_{i}\right) f(x)+o(f(x))$ and the calculations in (98) are replaced by

$$
\begin{aligned}
& V\left(f_{1}(x), \ldots, f_{n}(x)\right)=\prod_{1 \leq i<j \leq n}\left[\left(c_{j}-c_{i}\right) f(x)+o(f(x))\right] \\
= & \left(\prod_{1 \leq i<j \leq n}\left(c_{j}-c_{i}\right)\right) \cdot(f(x))^{n(n-1) / 2}+o\left(|f(x)|^{n(n-1) / 2}\right) \\
= & V\left(c_{1}, \ldots, c_{n}\right) \cdot(f(x))^{n(n-1) / 2}+o\left(|f(x)|^{n(n-1) / 2}\right),
\end{aligned}
$$

which is (81). Observe that these calculations start from the conclusions in part (II) valid under conditions in (75) which, in the present situation, are satisfied if " $c_{i} \neq 0 \forall i$ " $\left(f_{1} \asymp f_{2} \asymp \cdots \asymp f_{n}\right)$ or if " $c_{i} \neq 0,1 \leq i \leq n-1, c_{n}=0 "\left(f_{1} \asymp f_{2} \asymp \cdots \asymp f_{n-1} \gg f_{n}\right)$. We directly check this last case so that the reader may notice the difference with the case of more vanishing $c_{i}$ 's:

$$
\begin{aligned}
& V\left(c_{1} f(x)+o(f(x)), \ldots, c_{n-1} f(x)+o(f(x)), o(f(x))\right)= \\
= & \left|\begin{array}{cccc}
1 & \ldots & 1 & 1 \\
\left(c_{1} f(x)\right)^{i}[1+o(1)] & \ldots & \left(c_{n-1} f(x)\right)^{i}[1+o(1)] & o(f(x))
\end{array}\right|_{i=1, \ldots, n-1} \\
= & (f(x))^{n(n-1) / 2}\left|\begin{array}{cccc}
1 & \ldots & 1 & 1 \\
c_{1}^{i}+o(1) & \ldots & c_{n-1}^{i}+o(1) & 0^{i}+o(1)
\end{array}\right|_{i=1, \ldots, n-1}=\ldots
\end{aligned}
$$

(by the same reasoning as at the outset of the proof)

$\ldots=V\left(c_{1}, \ldots, c_{n-1}, 0\right) \cdot(f(x))^{n(n-1) / 2}+o(1)$.

But if two or more of the $c_{i}$ 's vanish then an independent proof is needed: see also Proposition 7 below. Suppose that:

$$
f_{i}(x)= \begin{cases}c_{i} f(x)+o(f(x)), c_{i} \neq 0, & i=1, \ldots, k,(k \leq n-2) \\ o(f(x)), & i=k+1, \ldots, n\end{cases}
$$

then:

$$
\begin{aligned}
& V\left(c_{1} f(x)+o(f(x)), \ldots, c_{k} f(x)+o(f(x)), o(f(x)), \ldots o(f(x))\right)= \\
= & \left|\begin{array}{cccccc}
1 & \ldots & 1 & 1 & \ldots & 1 \\
\left(c_{1} f(x)\right)^{i}[1+o(1)] & \ldots & \left(c_{k} f(x)\right)^{i}[1+o(1)] & o(f(x)) & \ldots & o(f(x))
\end{array}\right|_{i=1, \ldots, n-1} \\
= & (f(x))^{n(n-1) / 2}\left|\begin{array}{cccccc}
1 & \ldots & 1 & 1 & \ldots & 1 \\
c_{1}^{i}+o(1) & \ldots & c_{n-1}^{i}+o(1) & o(1) & \ldots & o(1)
\end{array}\right|_{i=1, \ldots, n-1}=o(1),
\end{aligned}
$$

having expanded the determinant with respect to the elements of the last column. Part (IV) is completely proved. The general assertion in (V) follows from combining (III) and (IV) as each factor in (97) satisfies one of the following relations:

either $f_{j}(x)-f_{i}(x) \sim-f_{i}(x) \quad$ or $\quad f_{j}(x)-f_{i}(x)=c_{i, j} f(x)+o(f(x)), x \rightarrow x_{0}$, 
with suitable (possibly zero) constants $c_{i, j}$ and suitable functions $f$, one for each group satisfying (80). And here are the calculations for the special case (87)-(88). Let us write out all the factors in the development of the Vandermondian as follows:

$$
\begin{aligned}
& V\left(f_{1}(x), \ldots, f_{m}(x), f_{m+1}(x), \ldots, f_{n}(x)\right)=(-1)^{n(n-1) / 2} \times \prod_{m+1 \leq i<j \leq n}\left(f_{i}-f_{j}\right) \times \\
& \times\left[\left(f_{1}-f_{2}\right) \cdots\left(f_{1}-f_{n}\right)\right] \cdot\left[\left(f_{2}-f_{3}\right) \cdots\left(f_{2}-f_{n}\right)\right] \cdots\left[\left(f_{m}-f_{m+1}\right) \cdots\left(f_{m}-f_{n}\right)\right] .
\end{aligned}
$$

In comparing with (59) notice the reverse order of each factor $\left(f_{i}-f_{j}\right)$. Now, as $x \rightarrow x_{0}$ :

$$
\begin{aligned}
& \prod_{m+1 \leq i<j \leq n}\left(f_{i}-f_{j}\right)=V\left(f_{n}(x), f_{n-1}(x), \ldots, f_{m+1}(x)\right) \stackrel{(81)}{=} \\
& =V\left(c_{n}, c_{n-1}, \ldots, c_{m+1}\right) \cdot(f(x))^{(n-m)(n-m-1) / 2}+o\left(|f(x)|^{(n-m)(n-m-1) / 2}\right) .
\end{aligned}
$$

In the first circumstance (88) it is obvious that the other $m$ groups of factors in square brackets in (104) are asymptotically equivalent respectively to

$$
\left(f_{1}(x)\right)^{n-1},\left(f_{2}(x)\right)^{n-2}, \ldots,\left(f_{m}(x)\right)^{n-m}
$$

and we get:

$$
\begin{aligned}
V\left(f_{1}(x), \ldots, f_{n}(x)\right) & =(-1)^{n(n-1) / 2} \cdot V\left(c_{n}, c_{n-1}, \ldots, c_{m+1}\right) \times \\
& \times(f(x))^{(n-m)(n-m-1) / 2} \cdot\left(\prod_{i=1}^{m}\left(f_{i}(x)\right)^{n-i}\right)+o(\ldots),
\end{aligned}
$$

whence (89) follows. In the second circumstance (88) we have:

$$
\begin{aligned}
\left(f_{1}-f_{2}\right) \cdots\left(f_{1}-f_{n}\right) & =\left(f_{1}-f_{2}\right) \cdots\left(f_{1}-f_{m}\right)\left(f_{1}-f_{m+1}\right) \cdots\left(f_{1}-f_{n}\right) \sim \\
& \sim(-1)^{n-m}\left(\prod_{i=m+1}^{n} c_{i}\right)(f(x))^{n-m}\left(f_{1}(x)\right)^{m-1} ;
\end{aligned}
$$

and analogously for the remaining groups of factors: the factor $\left(f_{1}(x)\right)^{m-1}$ in (108) being replaced successively by " $\left(f_{2}(x)\right)^{m-2}, \ldots,\left(f_{m-1}(x)\right)^{1}, 1$ ", while the constants and $(f(x))^{n-m}$ remain unchanged. At last we get:

$$
\begin{aligned}
V\left(f_{1}(x), \ldots, f_{n}(x)\right) & =(-1)^{[n(n-1) / 2]+m(n-m)} \cdot\left(\prod_{i=m+1}^{n} c_{i}\right)^{m} \cdot V\left(c_{n}, c_{n-1}, \ldots, c_{m+1}\right) \times \\
& \times(f(x))^{(n-m)(m+n-1) / 2}\left(\prod_{i=1}^{m-1}\left(f_{i}(x)\right)^{m-i}\right)+o(\ldots),
\end{aligned}
$$

whence (90) follows.

The result in Theorem 6-(IV) for some coinciding $c_{i}$ 's is included in the following

Proposition 7. (The case of rough asymptotic estimates). If $g_{1}, \ldots, g_{n}$ and $f_{1}, \ldots, f_{n}, n \geq 2$, are functions defined on a deleted neighborhood of $x_{0} \in \overline{\mathbb{R}}$ such that:

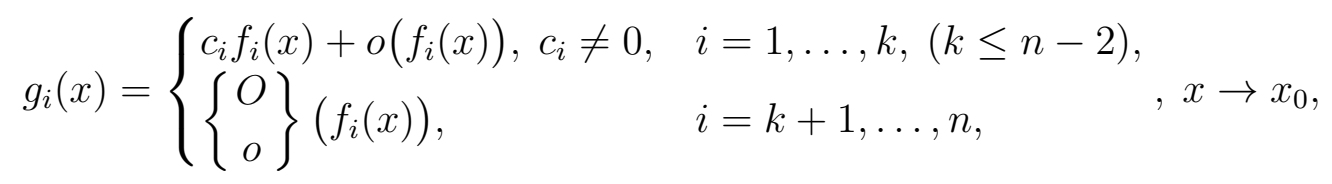


then relation (74) is replaced by the estimate:

$$
V\left(g_{1}(x), \ldots, g_{n}(x)\right)=\left\{\begin{array}{l}
O \\
o
\end{array}\right\}\left(\sum_{\left(p_{1}, \ldots, p_{n}\right) \in \mathcal{P}} \prod_{i=1}^{n}\left|f_{i}(x)\right|^{p_{i}}\right), x \rightarrow x_{0},
$$

where $\mathcal{P}$ denotes the set of all permutations of $(0,1, \ldots, n-1)$. This formula has in general a rather limited usefulness in so far as the assumptions on the $g_{i}$ 's do not imply any asymptotic hierarchy between the $f_{i}$ 's for $k+1 \leq i \leq n$. For $k=n-1$ the simplified relation

$$
V\left(g_{1}(x), \ldots, g_{n}(x)\right)=\left\{\begin{array}{c}
O \\
o
\end{array}\right\}\left(\prod_{i=1}^{n-1}\left|f_{i}(x)\right|^{n-i}\right), x \rightarrow x_{0}
$$

is included in Theorem 3-(II); and in the special case " $f_{i} \asymp f_{j} \forall i, j$ " we have a simplified relation as well:

$$
V\left(g_{1}(x), \ldots, g_{n}(x)\right)=\left\{\begin{array}{l}
O \\
o
\end{array}\right\}\left(\left|f_{1}(x)\right|^{n(n-1) / 2}\right), x \rightarrow x_{0} .
$$

Proof. Exactly as at the outset of the proof of Theorem 6 each term of the development of our determinant is a product of type

$$
\pm\left(\prod_{i=1}^{k}\left(f_{i}(x)\right)^{p_{i}}\right) \cdot\left\{\begin{array}{c}
O \\
o
\end{array}\right\}\left(\prod_{i=k+1}^{n}\left|f_{i}(x)\right|^{p_{i}}\right)+o\left(\prod_{i=1}^{n}\left|f_{i}(x)\right|^{p_{i}}\right)=\left\{\begin{array}{l}
O \\
o
\end{array}\right\}\left(\prod_{i=1}^{n}\left|f_{i}(x)\right|^{p_{i}}\right) .
$$

\section{Wronskians of Asymptotic Scales of Regularly- or Rapidly-Varying Functions}

This section contains the main results in this Part I of our work, namely the asymptotic behavior of Wronskians of $n$-tuples formed by functions with the same type of variation at $+\infty$ : either regular, smooth or rapid. The most meaningful results are obtained when the logarithmic derivatives of the $n$ functions are linked by a chain of asymptotic relations. We first state a strenghtened version of an elementary proposition highlighting the import that an inequality satisfied by the ratio of two logarithmic derivatives has on the asymptotic behavior of the ratio of the two given functions. This clasical result may be seen as a kind of L'Hospital's rule wherein ordinary derivatives are replaced by logarithmic derivatives.

Lemma 8. Let $f, g \in A C[T,+\infty)$ be such that:

$$
\left\{\begin{array}{l}
f(x), g(x)>0 \forall x ; \\
\text { each of the two functions } f^{\prime}, g^{\prime} \text { is almost everywhere strictly one-signed }(>0 \text { or }<0) ;
\end{array}\right.
$$

and

$$
\text { each of the two limits “ } \lim _{x \rightarrow+\infty} f(x), \lim _{x \rightarrow+\infty} g(x) ” \quad \text { is either zero or }+\infty \text {, }
$$

which is equivalent to stating that each of the two improper integrals

$$
\int^{+\infty} f^{\prime}(t) / f(t) \mathrm{d} t, \quad \int^{+\infty} g^{\prime}(t) / g(t) \mathrm{d} t \quad \text { diverges to either }+\infty \text { or }-\infty .
$$

Thesis. If ultimately

$$
\text { either } \quad 1<l_{1} \leq \frac{f^{\prime}(x)}{f(x)} / \frac{g^{\prime}(x)}{g(x)} \quad \text { or } \quad \frac{f^{\prime}(x)}{f(x)} / \frac{g^{\prime}(x)}{g(x)} \leq l_{2}<1,
$$


in particular, if

$$
\exists \lim _{x \rightarrow+\infty} \frac{f^{\prime}(x)}{f(x)} / \frac{g^{\prime}(x)}{g(x)} \equiv l \in \overline{\mathbb{R}} \backslash\{1\},
$$

then one of the two circumstances occur:

$$
f(x)=o(g(x)) \quad \text { or } \quad g(x)=o(f(x)), x \rightarrow+\infty .
$$

More precisely, in case of condition (119) we have the following tables:

(I) If $1<l \leq+\infty$ then, as $x \rightarrow+\infty$ :

$$
\left\{\begin{array}{lll}
\operatorname{sign} f^{\prime}=\operatorname{sign} g^{\prime}=+1 & \Rightarrow & f(x) \gg g(x) ; \\
\operatorname{sign} f^{\prime}=\operatorname{sign} g^{\prime}=-1 & \Rightarrow & f(x) \ll g(x) .
\end{array}\right.
$$

(II) If $-\infty \leq l<1$ then, as $x \rightarrow+\infty$ :

$$
\left\{\begin{array}{lll}
\operatorname{sign} f^{\prime}=-\operatorname{sign} g^{\prime}=+1 & \Rightarrow & f(x)=+\infty(1), g(x)=o(1) ; \\
\operatorname{sign} f^{\prime}=-\operatorname{sign} g^{\prime}=-1 & \Rightarrow & f(x)=o(1), \quad g(x)=+\infty(1) ; \\
\operatorname{sign} f^{\prime}=\operatorname{sign} g^{\prime}=+1 & \Rightarrow & f(x) \ll g(x) ; \\
\operatorname{sign} f^{\prime}=\operatorname{sign} g^{\prime}=-1 & \Rightarrow & f(x) \gg g(x) .
\end{array}\right.
$$

(III) If

$$
\left\{\begin{array}{l}
f^{\prime}(x) / f(x)=\phi(x)\left[c_{1}+o(1)\right] \\
g^{\prime}(x) / g(x)=\phi(x)\left[c_{2}+o(1)\right]
\end{array}, x \rightarrow+\infty ; c_{1}<c_{2} ; \phi>0 ;\right.
$$

then $f(x) \ll g(x), x \rightarrow+\infty$.

Proof. The argument is based on representations:

$$
\begin{gathered}
f(x)=f(T) \cdot \exp \left(\int_{T}^{x} f^{\prime}(t) / f(t) \mathrm{d} t\right) ; g(x)=g(T) \cdot \exp \left(\int_{T}^{x} g^{\prime}(t) / g(t) \mathrm{d} t\right) ; \\
\frac{f(x)}{g(x)}=\frac{f(T)}{g(T)} \cdot \exp \left(\int_{T}^{x}\left[\frac{f^{\prime}(t)}{f(t)}-\frac{g^{\prime}(t)}{g(t)}\right] \mathrm{d} t\right) .
\end{gathered}
$$

The equivalence between (116) and (117) follows at once from (124a) and the claims when $f^{\prime}$ and $g^{\prime}$ have opposite signs are trivial consequences of (115)-(116). And also the proof under the strong condition (119) is quite immediate as, in this case, one of the three circumstances occur:

$$
\left(f^{\prime}(x) / f(x)\right)=\left\{\begin{array}{l}
-\infty\left(g^{\prime}(x) / g(x)\right) \\
\left(g^{\prime}(x) / g(x)\right) \cdot[l+o(1)], x \rightarrow+\infty \\
+\infty\left(g^{\prime}(x) / g(x)\right)
\end{array}\right.
$$

and the assertions in (I)-(II), for $l \geq 0$, as well as the inference in (III), directly follow from (124b). Under the first weaker conditions in (118) it follows that "sign $f^{\prime}=\operatorname{sign} g^{\prime}$ ultimately a.e." and

$$
0<\frac{g^{\prime}(x) / g(x)}{f^{\prime}(x) / f(x)} \leq \frac{1}{l_{1}}<1 \quad \text { hence } \quad 1-\frac{g^{\prime}(x) / g(x)}{f^{\prime}(x) / f(x)} \geq 1-\frac{1}{l_{1}}>0 .
$$

Replaging into the identity:

$$
M(x):=\frac{f^{\prime}(x)}{f(x)}-\frac{g^{\prime}(x)}{g(x)} \equiv \frac{f^{\prime}(x)}{f(x)} \cdot\left[1-\frac{g^{\prime}(x) / g(x)}{f^{\prime}(x) / f(x)}\right],
$$


we get:

$$
\begin{aligned}
& \left\{\begin{array}{l}
f^{\prime}(x) / f(x)>0 \Rightarrow M(x) \geq\left(1-\frac{1}{l_{1}}\right)\left(f^{\prime}(x) / f(x)\right)>0 \text { a. e. } \stackrel{b y}{\Rightarrow}(117) \\
\Rightarrow \int^{+\infty} M=+\infty \Rightarrow f(x) / g(x) \rightarrow+\infty
\end{array}\right. \\
& \left\{\begin{array}{l}
f^{\prime}(x) / f(x)<0 \Rightarrow M(x) \leq\left(1-\frac{1}{l_{1}}\right)\left(f^{\prime}(x) / f(x)\right)<0 \text { a. e. } \stackrel{b y}{\Rightarrow}(117) \\
\Rightarrow \int^{+\infty} M=-\infty \Rightarrow f(x) / g(x) \rightarrow 0 .
\end{array}\right.
\end{aligned}
$$

Assuming the second weaker conditions in (118) and "sign $f^{\prime}=\operatorname{sign} g^{\prime}$ " then, by inverting the roles of $f$ and $g$, the above argument may be reapplied.

In the assumptions of Theorems 9,10 we write " $\phi_{i} \in C^{n-1}[T,+\infty)$ " to make the statement immediately grasped; nothing is changed under the weaker assumptions " $\phi_{i} \in A C^{n-2}[T,+\infty)$ ", i.e. $\phi_{i}^{(n-2)}$ absolutely continuous, provided that the asymptotic relations are read "with $x$ running through the points wherein the $(n-1)$-th derivatives exist as finite numbers".

Theorem 9. (Principal parts of Wronskians of smoothly-varying functions). For $i=1, \ldots, n$ let $\phi_{i} \in C^{n-1}[T,+\infty), \phi_{i}(x) \neq 0$ for $x$ large enough, and

$$
\phi_{i}^{(k)}(x) / \phi_{i}(x)=x^{-k}\left[\left(a_{i}\right)^{\underline{k}}+o(1)\right], x \rightarrow+\infty ; 1 \leq k \leq n-1 ; 1 \leq i \leq n ;
$$

where $a_{i}$ are any real numbers, i.e. $\phi_{i}$ is smoothly (in particular, regularly) varying at $+\infty$ of order $n-1$ and index $\alpha_{i}$ (Definitions 1,2). Then for the Wronskian of the $\phi_{i}$ 's the following relation holds true:

$$
W\left(\phi_{1}(x), \ldots, \phi_{n}(x)\right)=\left(\prod_{i=1}^{n} \phi_{i}(x)\right) \cdot x^{-n(n-1) / 2} \cdot\left[V\left(a_{1}, \ldots, a_{n}\right)+o(1)\right], x \rightarrow+\infty .
$$

If $a_{1}>a_{2}>\cdots>a_{n}$ then $\left(\phi_{1}, \ldots, \phi_{n}\right)$ is an asymptotic scale at $+\infty$ and

$$
W\left(\phi_{1}(x), \ldots, \phi_{n}(x)\right) \sim V\left(a_{1}, \ldots, a_{n}\right) \cdot\left(\prod_{i=1}^{n} \phi_{i}(x)\right) \cdot x^{-n(n-1) / 2}, x \rightarrow+\infty .
$$

Proof. Using (130) into the right-hand side of (10) we get:

$$
\begin{aligned}
& W\left(\phi_{1}(x), \ldots, \phi_{n}(x)\right)= \\
& =\left(\prod_{i=1}^{n} \phi_{i}(x)\right) \cdot\left|\begin{array}{ccc}
1 & \ldots & 1 \\
x^{-1}\left[a_{1}+o(1)\right] & \ldots & x^{-1}\left[a_{n}+o(1)\right] \\
x^{-2}\left[\left(a_{1}\right)^{2}+o(1)\right] & \ldots & x^{-2}\left[\left(a_{n}\right)^{2}+o(1)\right] \\
\ldots & \ldots & \ldots \\
x^{-(n-1)}\left[\left(a_{1}\right)^{\frac{n-1}{2}}+o(1)\right] & \ldots & x^{-(n-1)}\left[\left(a_{n}\right)^{\frac{n-1}{n}}+o(1)\right]
\end{array}\right|= \\
& =\left(\prod_{i=1}^{n} \phi_{i}(x)\right) \cdot x^{-1-2-\cdots-(n-1)}\left|\begin{array}{ccc}
1 & \ldots & 1 \\
a_{1}+o(1) & \ldots & a_{n}+o(1) \\
\left(a_{1}\right)^{2}+o(1) & \ldots & \left(a_{n}\right)^{2}+o(1) \\
\ldots & \ldots & \ldots \\
\left(a_{1}\right)^{\frac{n-1}{2}}+o(1) & \ldots & \left(a_{n}\right)^{\frac{n-1}{2}}+o(1)
\end{array}\right| .
\end{aligned}
$$


In the last determinant we apply the elementary procedure used to prove the identity (66): from the third row we subtract the second row, then from the fourth row a suitable linear combination of the preceding rows and so on. By so doing we get:

$$
\begin{gathered}
W\left(\phi_{1}(x), \ldots, \phi_{n}(x)\right)=\left(\prod_{i=1}^{n} \phi_{i}(x)\right) \cdot x^{-n(n-1) / 2} \cdot\left|\begin{array}{ccc}
1 & \ldots & 1 \\
a_{1}+o(1) & \ldots & a_{n}+o(1) \\
a_{1}^{2}+o(1) & \ldots & a_{n}^{2}+o(1) \\
\ldots & \ldots & \ldots \\
a_{1}^{n-1}+o(1) & \ldots & a_{n}^{n-1}+o(1)
\end{array}\right| \\
\stackrel{b y}{=}\left(\prod_{i=1}^{n} \phi_{i}(x)\right) \cdot x^{-n(n-1) / 2} \cdot\left[V\left(a_{1}, \ldots, a_{n}\right)+o(1)\right] .
\end{gathered}
$$

If $a_{1}>\cdots>a_{n}$ then $\phi_{i}^{\prime}(x) / \phi_{i}(x)=x^{-1}\left[a_{i}+o(1)\right]$ and Lemma 8-(III) implies that $\left(\phi_{1}, \ldots, \phi_{n}\right)$ is an asymptotic scale at $+\infty$.

Theorem 10. (Principal parts of Wronskians of rapidly-varying functions). For $i=1, \ldots$, n let $\phi_{i} \in$ $C^{n-1}[T,+\infty)$ be rapidly varying at $+\infty$ of order $n-1$ and in the strong sense of Definition 3 , i.e.

$$
\left\{\begin{array}{l}
\phi^{(k)}(x) \neq 0 \forall x \text { large enough and } 0 \leq k \leq n ; \\
\left.\lim _{x \rightarrow+\infty} \phi_{i}^{\prime}(x) / \phi_{i}(x)= \pm \infty \quad \text { (the sign } \pm \text { depending on } i\right) \\
\phi_{i}^{(k)}(x) / \phi_{i}(x) \sim\left(\phi_{i}^{\prime}(x) / \phi_{i}(x)\right)^{k}, x \rightarrow+\infty ; 2 \leq k \leq n-1,1 \leq i \leq n .
\end{array}\right.
$$

Then: (I) For the Wronskian of the $\phi_{i}$ 's the following relation holds true:

$$
\begin{aligned}
& W\left(\phi_{1}(x), \ldots, \phi_{n}(x)\right)=\left(\prod_{i=1}^{n} \phi_{i}(x)\right) \times \\
& \times\left[V\left(\frac{\phi_{1}^{\prime}(x)}{\phi_{1}(x)}, \ldots, \frac{\phi_{n}^{\prime}(x)}{\phi_{n}(x)}\right)+o\left(\sum_{\left(p_{1}, \ldots, p_{n}\right) \in \mathcal{P}} \prod_{i=1}^{n}\left|\frac{\phi_{i}^{\prime}(x)}{\phi_{i}(x)}\right|^{p_{i}}\right)\right], x \rightarrow+\infty,
\end{aligned}
$$

where $\mathcal{P}$ denotes the set of all permutations of $(0,1, \ldots, n-1)$.

(II) Under the special further assumption that for each index $i$ :

either $\phi_{i}^{\prime}(x) / \phi_{i}(x) \gg \phi_{i+1}^{\prime}(x) / \phi_{i+1}(x) \quad$ or $\quad \phi_{i}^{\prime}(x) / \phi_{i}(x) \asymp \phi_{i+1}^{\prime}(x) / \phi_{i+1}(x), x \rightarrow+\infty$,

the last symbol being defined in (18), we have the rough estimate:

$$
W\left(\phi_{1}(x), \ldots, \phi_{n}(x)\right)=O\left(\left(\prod_{i=1}^{n} \phi_{i}(x)\right) \cdot\left(\prod_{i=1}^{n-1}\left|\frac{\phi_{i}^{\prime}(x)}{\phi_{i}(x)}\right|^{n-i}\right)\right), x \rightarrow+\infty,
$$

noticing the lack of $\phi_{n}^{\prime}(x) / \phi_{n}(x)$, one of the logarithmic derivatives $\phi_{i}^{\prime}(x) / \phi_{i}(x)$ with the lowest growth-order.

(III) Under the special further assumption that:

$$
\frac{\phi_{1}^{\prime}(x)}{\phi_{1}(x)} \gg \cdots \gg \frac{\phi_{n}^{\prime}(x)}{\phi_{n}(x)}, x \rightarrow+\infty
$$

there exists a permutation $\left(\phi_{i_{1}}, \ldots, \phi_{i_{n}}\right)$ forming an asymptotic scale at $+\infty$ and (136) is replaced by the precise relation:

$$
W\left(\phi_{1}(x), \ldots, \phi_{n}(x)\right) \sim(-1)^{n(n-1) / 2}\left(\prod_{i=1}^{n} \phi_{i}(x)\right) \cdot\left(\prod_{i=1}^{n-1}\left(\frac{\phi_{i}^{\prime}(x)}{\phi_{i}(x)}\right)^{n-i}\right), x \rightarrow+\infty .
$$


(IV) Under the special further assumption that:

$$
\phi_{i}^{\prime}(x) / \phi_{i}(x)=c_{i} \phi(x)+o(\phi(x)), x \rightarrow+\infty, 1 \leq i \leq n,
$$

for some fixed function $\phi$ and arbitrary constants $c_{i}$ (possibly $c_{i}=0$ for some $i$ ), (136) is replaced by:

$$
W\left(\phi_{1}(x), \ldots, \phi_{n}(x)\right)=\left(\prod_{i=1}^{n} \phi_{i}(x)\right) \cdot(\phi(x))^{n(n-1) / 2} \cdot\left[V\left(c_{1}, \ldots, c_{n}\right)+o(1)\right], x \rightarrow+\infty .
$$

With the restriction " $c_{1}>\cdots>c_{n}$ " then $\left(\phi_{1}, \ldots, \phi_{n}\right)$ is an asymptotic scale at $+\infty$ and

$$
W\left(\phi_{1}(x), \ldots, \phi_{n}(x)\right) \sim V\left(c_{1}, \ldots, c_{n}\right) \cdot\left(\prod_{i=1}^{n} \phi_{i}(x)\right) \cdot(\phi(x))^{n(n-1) / 2}, x \rightarrow+\infty .
$$

If $c_{1}<\cdots<c_{n}$ then $\left(\phi_{n}, \ldots, \phi_{1}\right)$ is an asymptotic scale at $+\infty$ and the foregoing relation is still true. The special case wherein all the $c_{i}$ 's coincide and (141) are replaced by two-term asymptotic expansions:

$$
\phi_{i}^{\prime}(x) / \phi_{i}(x)=\phi(x)+c_{i} \psi_{i}(x)+o\left(\psi_{i}(x)\right), x \rightarrow+\infty, 1 \leq i \leq n,
$$

for some fixed function $\phi$ and arbitrary constants $c_{i}$, is not particularly meaningful in the present context as it refers to the case wherein there is a factor common to all the $\phi_{i}$ 's and which may obviously be factored out in the Wronskian (by Hesse-Christoffel's identity).

(V) Suppose that the functions $\phi_{i}^{\prime}(x) / \phi_{i}(x)$ may be grouped in several ordered $i_{k}$-tuples each of which either forms an asymptotic scale at $+\infty$ or contains elements with the same growth-order in the sense of (141), more precisely:

$$
\begin{aligned}
\frac{\phi_{1}^{\prime}(x)}{\phi_{1}(x)} & \sim c_{1} \frac{\phi_{2}^{\prime}(x)}{\phi_{2}(x)} \sim \cdots \sim c_{i_{1}-1} \frac{\phi_{i_{1}}^{\prime}(x)}{\phi_{i_{1}}(x)} \gg \frac{\phi_{i_{1}+1}^{\prime}(x)}{\phi_{i_{1}+1}(x)} \gg \cdots \gg \frac{\phi_{i_{2}}^{\prime}(x)}{\phi_{i_{2}}(x)} \sim \\
& \sim d_{1} \frac{\phi_{i_{2}+1}^{\prime}(x)}{\phi_{i_{2}+1}(x)} \sim d_{2} \frac{\phi_{i_{2}+2}^{\prime}(x)}{\phi_{i_{2}+2}(x)} \sim \cdots \sim d_{i_{3}-i_{2}} \frac{\phi_{i_{3}}^{\prime}(x)}{\phi_{i_{3}}(x)} \gg \cdots \gg \ldots,
\end{aligned}
$$

where

$$
i_{1}+i_{2}+\cdots+i_{k}=n ; \quad c_{1}>\cdots>c_{i_{1}-1} ; \quad d_{1}>\cdots>d_{i_{3}-i_{2}} \quad \text { and so on. }
$$

Then a suitable permutation of $\left(\phi_{1}, \ldots, \phi_{n}\right)$ forms an asymptotic scale at $+\infty$ and (136) takes the form:

$$
W\left(\phi_{1}(x), \ldots, \phi_{n}(x)\right) \sim C \cdot\left(\prod_{i=1}^{n} \phi_{i}(x)\right) \cdot\left(\prod_{i=1}^{n-1}\left(\frac{\phi_{i}^{\prime}(x)}{\phi_{i}(x)}\right)^{n-i}\right), x \rightarrow+\infty,
$$

where $C$ is a nonzero constant solely dependent on the various constants $c_{i}, d_{i}, \ldots$ appearing in the chain (145) and where each group of asymptotically-equivalent factors $\left(\phi_{i}^{\prime}(x) / \phi_{i}(x)\right)^{n-i}$ may be obviously replaced by only one factor with the suitable exponent, if so desired.

(VI) If the n-tuple $\left(\phi_{1}, \ldots, \phi_{n}\right)$ contains one constant, say $\phi_{k}(x) \equiv c \neq 0$, and if the remaining functions satisfy (135) we may obviously apply the foregoing results to the reduced Wronskian:

$$
W\left(\phi_{1}(x), \ldots, \phi_{n}(x)\right)=(-1)^{k-1} c \cdot W\left(\phi_{1}^{\prime}(x), \ldots, \phi_{k-1}^{\prime}(x), \phi_{k+1}^{\prime}(x), \ldots, \phi_{n}^{\prime}(x)\right) .
$$

The general case wherein one or more of the $\phi_{k}$ 's are smoothly varying will be the main problem treated in Part II of this work. 
Proof. (I) Replacing the asymptotic relations (135) into the right-hand side of (10) we get:

$$
\begin{aligned}
& W\left(\phi_{1}(x), \ldots, \phi_{n}(x)\right)=\left(\prod_{i=1}^{n} \phi_{i}(x)\right) \times \\
& \times\left|\begin{array}{ccc}
1 & \ldots & 1 \\
\phi_{1}^{\prime}(x) / \phi_{1}(x) & \ldots & \phi_{n}^{\prime}(x) / \phi_{n}(x) \\
\left(\phi_{1}^{\prime}(x) / \phi_{1}(x)\right)^{2}[1+o(1)] & \ldots & \left(\phi_{n}^{\prime}(x) / \phi_{n}(x)\right)^{2}[1+o(1)] \\
\vdots & & \vdots \\
\left(\phi_{1}^{\prime}(x) / \phi_{1}(x)\right)^{n-1}[1+o(1)] & \ldots & \left(\phi_{n}^{\prime}(x) / \phi_{n}(x)\right)^{n-1}[1+o(1)]
\end{array}\right|
\end{aligned}
$$

whence, by (74), (136) follows. (II) follows from (77). (III) and (IV): Under condition (139) Lemma 8 implies that:

$$
\lim _{x \rightarrow+\infty} \phi_{i}(x) / \phi_{j}(x)=\text { either } 0 \text { or } \pm \infty \forall i, j,
$$

and so a suitable rearrangement of the $\phi_{i}^{\prime}$ s forms an asymptotic scale. Relations in (140) and (142) follow by applying respectively (79) or (81). Part (V) follows from (III) and (IV).

The precise asymptotic relations in (143) and (147) require that the indexes $a_{i}, c_{i}$ are distinct. For a scale involving smoothly-varying functions $\phi_{i}$, all with the same index $\alpha$, we may use factorization $\phi_{i}(x)=x^{\alpha} \mathcal{L}_{i}(x)$ and identity (25) to write:

$$
W\left(\phi_{1}(x), \ldots, \phi_{n}(x)\right)=x^{n \alpha} \cdot W\left(\mathcal{L}_{1}(x), \ldots, \mathcal{L}_{n}(x)\right) \text { with } \mathcal{L}_{i} \text { slowly varying at }+\infty,
$$

and the problem reduces to studying the Wronskian of slowly-varying functions. However a (seemingly) simple example such as the Wronskian $W\left(\ell_{1}(x), \ldots, \ell_{n}(x)\right)$ shows (after many trials) that the previous methods or variants of them do not work. An indirect approach will be suggested in Part II.

\section{Examples Involving Asymptotic Scales}

All the following are meant as asymptotic scales as $x \rightarrow+\infty$ if not explicitly stated.

Example 5.1. The general real-power asymptotic scale. For the scale

$$
(\phi(x))^{\alpha_{1}} \gg(\phi(x))^{\alpha_{2}} \gg \cdots \gg(\phi(x))^{\alpha_{n}} ; \alpha_{1}>\alpha_{2}>\cdots>\alpha_{n},
$$

where $\lim _{x \rightarrow+\infty} \phi(x)=+\infty$ and $\phi$ is $(n-1)$-times differentiable, we have the exact formula (68) for each $n \geq 2$ and all $x$ such that $\phi(x)>0$ :

$$
W\left((\phi(x))^{\alpha_{1}}, \ldots,(\phi(x))^{\alpha_{n}}\right)=\left(\prod_{1 \leq i<j \leq n}\left(\alpha_{j}-\alpha_{i}\right)\right) \cdot(\phi(x))^{\alpha_{1}+\cdots+\alpha_{n}-[n(n-1) / 2]} \cdot\left(\phi^{\prime}(x)\right)^{n(n-1) / 2}
$$

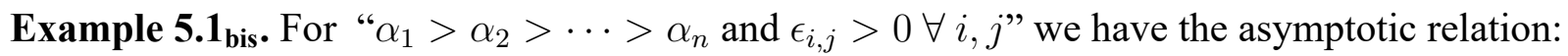

$$
\left\{\begin{array}{l}
W\left(x^{\alpha_{1}}+\sum_{i=1}^{m} c_{1, i} x^{\alpha_{1}-\epsilon_{1, i}}, x^{\alpha_{2}}+\sum_{i=1}^{m} c_{2, i} x^{\alpha_{2}-\epsilon_{2, i}}, \ldots, x^{\alpha_{n}}+\sum_{i=1}^{m} c_{n, i} x^{\alpha_{n}-\epsilon_{n, i}}\right) \sim \\
\sim\left(\prod_{1 \leq i<j \leq n}\left(\alpha_{j}-\alpha_{i}\right)\right) \cdot x^{\alpha_{1}+\alpha_{2}+\cdots+\alpha_{n}-[n(n-1) / 2]}, x \rightarrow+\infty,\left(\text { any constants } c_{k, i}\right) .
\end{array}\right.
$$

Example 5.1 $1_{\text {ter. }}$ For " $\alpha_{1}>\alpha_{2}>\cdots>\alpha_{n}, \epsilon_{i}>0$ and $b_{i} \in \mathbb{R} \forall i$ " we have the asymptotic relation:

$$
\left\{\begin{array}{l}
W\left(x^{\alpha_{1}}+x^{\alpha_{1}-\epsilon_{1}}(\log x)^{b_{1}}, x^{\alpha_{2}}+x^{\alpha_{2}-\epsilon_{2}}(\log x)^{b_{2}}, \ldots, x^{\alpha_{n}}+x^{\alpha_{n}-\epsilon_{n}}(\log x)^{b_{n}}\right) \sim \\
\sim\left(\prod_{1 \leq i<j \leq n}\left(\alpha_{j}-\alpha_{i}\right)\right) \cdot x^{\alpha_{1}+\alpha_{2}+\cdots+\alpha_{n}-[n(n-1) / 2]}, x \rightarrow+\infty
\end{array}\right.
$$


Example 5.2. Power-logarithmic asymptotic scales:

$$
\begin{aligned}
& x^{\alpha_{1}}(\log x)^{b_{1}} \gg x^{\alpha_{2}}(\log x)^{b_{2}} \gg \cdots \gg x^{\alpha_{n}}(\log x)^{b_{n}} ; \alpha_{1}>\alpha_{2}>\cdots>\alpha_{n} ; b_{i} \in \mathbb{R} ; \\
& \left\{\begin{array}{l}
W\left(x^{\alpha_{1}}(\log x)^{b_{1}}, \ldots, x^{\alpha_{n}}(\log x)^{b_{n}}\right) \sim \\
\sim\left(\prod_{1 \leq j<i \leq n}\left(\alpha_{i}-\alpha_{j}\right)\right) \cdot x^{\alpha_{1}+\alpha_{2}+\cdots+\alpha_{n}-[n(n-1) / 2]}(\log x)^{b_{1}+b_{2}+\cdots+b_{n}}, x \rightarrow+\infty .
\end{array}\right.
\end{aligned}
$$

Example 5.3. A more general power-logarithmic asymptotic scale. For the scale

$$
x^{\alpha_{1}} \mathcal{L}_{1}(x) \gg x^{\alpha_{2}} \mathcal{L}_{2}(x) \gg \cdots \gg x^{\alpha_{n}} \mathcal{L}_{n}(x) ; \alpha_{1}>\alpha_{2}>\cdots>\alpha_{n},
$$

where each $\mathcal{L}_{i}$ is a function of type:

$$
\mathcal{L}_{i}(x):=\left(\prod_{k=1}^{n_{i}}\left(\ell_{k}(x)\right)^{b_{k, i}}\right) \cdot\left(\prod_{k=1}^{m_{i}} \exp \left(c_{i}(\log x)^{d_{i}}\right)\right) ; \quad b_{k, i}, c_{i} \in \mathbb{R} ; 0<d_{i}<1,
$$

we have the asymptotic relation:

$$
\left\{\begin{array}{l}
W\left(x^{\alpha_{1}} \mathcal{L}_{1}(x), \ldots, x^{\alpha_{n}} \mathcal{L}_{n}(x)\right) \sim \\
\sim\left(\prod_{1 \leq j<i \leq n}\left(\alpha_{i}-\alpha_{j}\right)\right) \cdot x^{\alpha_{1}+\alpha_{2}+\cdots+\alpha_{n}-[n(n-1) / 2]} \cdot\left(\prod_{i=1}^{n} \mathcal{L}_{i}(x)\right), x \rightarrow+\infty .
\end{array}\right.
$$

All of the above relations for the Wronskians obviously become simpler for the special choices of $\alpha_{i}$ such that: $\alpha_{1}+\alpha_{2}+\cdots+\alpha_{n}=n(n-1) / 2$.

Example 5.4. An "exponentials-of-powers" asymptotic scale. (i) For the scale

$$
\exp \left(c_{1} x^{d_{1}}\right) \gg \exp \left(c_{2} x^{d_{2}}\right) \gg \cdots \gg \exp \left(c_{n} x^{d_{n}}\right), x \rightarrow+\infty,\left(c_{i} \neq 0, d_{i}>0 \forall i\right),
$$

we are supposing one of the following set of conditions:

$$
\begin{aligned}
& c_{i}>0 \forall i \text { and } \quad d_{1}>d_{2}>\cdots>d_{n}>0 ; \\
& c_{i}<0 \forall i \text { and } 0<d_{1}<d_{2}<\cdots<d_{n} ;
\end{aligned}
$$

Here we have $\phi_{i}^{\prime}(x) / \phi_{i}(x)=c_{i} d_{i} x^{d_{i}-1}$, and Theorem 10-(III) applies so that we get the following asymptotic relation:

$$
\begin{aligned}
& W\left(\left\{\exp \left(c_{i} x^{d_{i}}\right)\right\}_{i=1, \ldots, n}\right) \sim(-1)^{n(n-1) / 2} \exp \left(\sum_{i=1}^{n} c_{i} x^{d_{i}}\right) \cdot\left(\prod_{i=1}^{n-1}\left(c_{i} d_{i}\right)^{n-i} x^{\left(d_{i}-1\right)(n-i)}\right) \\
& =(-1)^{n(n-1) / 2}\left(\prod_{i=1}^{n-1}\left(c_{i} d_{i}\right)^{n-i}\right) \cdot x^{M} \cdot \exp \left(\sum_{i=1}^{n} c_{i} x^{d_{i}}\right), x \rightarrow+\infty,
\end{aligned}
$$

where:

$$
M \equiv M\left(n, d_{i}\right):=(n-1)\left(d_{1}-1\right)+(n-2)\left(d_{2}-1\right)+\cdots+2\left(d_{n-2}-1\right)+\left(d_{n-1}-1\right) .
$$

(ii) Under conditions:

$$
\left\{\begin{array}{l}
c_{1}, \ldots, c_{k}>0 ; c_{k+1}, \ldots, c_{n}<0 \\
d_{1}>d_{2}>\cdots>d_{k}>0 ; 0<d_{k+1}<d_{k+2}<\cdots<d_{n}
\end{array}\right.
$$


we have again an asymptotic scale at $+\infty$ and the logarithmic derivatives may be ordered as in Theorem 10-(V); hence in the preceding asymptotic relation (164) the comparison function remains unchanged whereas the constant factor $(-1)^{n(n-1) / 2} \prod_{i=1}^{n-1}\left(c_{i} d_{i}\right)^{n-i}$ is replaced by a suitable nonzero constant. For instance, if " $d_{1}>d_{2}>\cdots>d_{k}>d_{n}>d_{n+1}>\cdots>d_{k+1}>0$ " then relation (164) is true if referred to the Wronskian

$$
W\left(\exp \left(c_{1} x^{d_{1}}\right), \ldots, \exp \left(c_{k} x^{d_{k}}\right), \exp \left(c_{n} x^{d_{n}}\right), \exp \left(c_{n-1} x^{d_{n-1}}\right), \ldots, \exp \left(c_{k+1} x^{d_{k+1}}\right)\right) ;
$$

and, more generally, if no two $d_{i}$ 's coincide then they may be reordered in a decreasing sequence " $d_{i_{1}}>\cdots>d_{i_{n}}$ " and relation (164) is true if referred to the Wronskian $W\left(\left\{\exp \left(c_{i_{j}} x^{d_{i_{j}}}\right)_{j=1, \ldots, n}\right\}\right)$.

If $n=2 m, m \geq 2$, and " $d_{i}=d_{2 m-i+1}, 1 \leq i \leq 2 m$," then the logarithmic derivatives may be grouped in $m$ ordered pairs according to the following rough scheme:

$$
c_{1} d_{1} x^{d_{1}-1} \asymp c_{2 m} d_{1} x^{d_{1}-1} \gg c_{2} d_{2} x^{d_{2}-1} \asymp c_{2 m-1} d_{2} x^{d_{2}-1} \gg \cdots \gg c_{m} d_{m} x^{d_{m}-1} \asymp c_{m+1} d_{m} x^{d_{m}-1}
$$

where, obviously, each relation $\asymp$ may be replaced by the relation $\sim$ after inserting the proper constants.

Example 5.5. Cases of conciding exponents. (i) For the scale

$$
\left\{\begin{array}{l}
\exp \left(c_{1} x^{d}\right) \gg \exp \left(c_{2} x^{d}\right) \gg \cdots \gg \exp \left(c_{n} x^{d}\right), x \rightarrow+\infty, \\
d>0, c_{1}>\cdots>c_{n}, c_{i} \neq 0 \forall i,
\end{array}\right.
$$

we have $\phi_{i}^{\prime}(x) / \phi_{i}(x)=c_{i} d x^{d-1} \forall i$, and Theorem 10-(IV) yields the relation as $x \rightarrow+\infty$ :

$W\left(\exp \left(c_{1} x^{d}\right), \ldots, \exp \left(c_{n} x^{d}\right)\right) \sim V\left(c_{1}, \ldots, c_{n}\right) \cdot\left(d x^{d-1}\right)^{n(n-1) / 2} \cdot \exp \left[\left(c_{1}+\cdots+c_{n}\right) x^{d}\right]$.

As special cases (under the same restrictions on the $c_{i}$ 's) we have:

$$
\begin{gathered}
W\left(e^{c_{1} x}, \ldots, e^{c_{n} x}\right) \sim V\left(c_{1}, \ldots, c_{n}\right) \cdot e^{\left(c_{1}+c_{2}+\cdots+c_{n}\right) x}, x \rightarrow+\infty ; \\
W\left(e^{c_{1} x^{2}}, \ldots, e^{c_{n} x^{2}}\right) \sim V\left(c_{1}, \ldots, c_{n}\right) \cdot(2 x)^{n(n-1) / 2} \cdot e^{\left(c_{1}+c_{2}+\cdots+c_{n}\right) x^{2}}, x \rightarrow+\infty .
\end{gathered}
$$

(ii) For the scale

$$
\exp \left(c_{1} x^{d_{1}}\right) \gg \cdots \gg \exp \left(c_{m} x^{d_{m}}\right) \gg \exp \left(c_{m+1} x^{d_{m}}\right) \gg \cdots \gg \exp \left(c_{n} x^{d_{m}}\right), x \rightarrow+\infty,
$$

where:

$$
\left\{\begin{array}{l}
1 \leq m \leq n-2 ; d_{i} \text { and } c_{i} \text { satisfy either }(162) \text { or }(163) \text { for } 1 \leq i \leq m+1 \\
c_{m+1}>\cdots>c_{n}, c_{i} \neq 0
\end{array}\right.
$$

we have:

$$
\left\{\begin{array}{l}
\phi_{i}^{\prime}(x) / \phi_{i}(x)=c_{i} d_{i} x^{d_{i}-1}, 1 \leq i \leq m+1 \\
\phi_{1}^{\prime}(x) / \phi_{1}(x) \gg \cdots \gg \phi_{m}^{\prime}(x) / \phi_{m}(x) \gg \phi_{m+1}^{\prime}(x) / \phi_{m+1}(x) \\
\phi_{i}^{\prime}(x) / \phi_{i}(x) \sim c_{i}\left(d_{m} x^{d_{m}-1}\right), m+1 \leq i \leq n
\end{array}\right.
$$

We now estimate the determinant $V\left(\frac{\phi_{1}^{\prime}(x)}{\phi_{1}(x)}, \ldots, \frac{\phi_{n}^{\prime}(x)}{\phi_{n}(x)}\right)$ as in Theorem 6-(V), formula (89):

$$
\begin{aligned}
& V\left(\frac{\phi_{1}^{\prime}(x)}{\phi_{1}(x)}, \ldots, \frac{\phi_{n}^{\prime}(x)}{\phi_{n}(x)}\right) \sim(-1)^{n(n-1) / 2} \cdot V\left(c_{n}, c_{n-1}, \ldots, c_{m+1}\right) \times \\
& \times\left(d_{m} x^{d_{m}-1}\right)^{(n-m)(n-m-1) / 2} \cdot \prod_{i=1}^{m}\left(c_{i} d_{i} x^{d_{i}-1}\right)^{n-i}= \\
& =(-1)^{n(n-1) / 2} \cdot V\left(c_{n}, c_{n-1}, \ldots, c_{m+1}\right) \cdot\left(d_{m}\right)^{(n-m)(n-m-1) / 2} \cdot \prod_{i=1}^{m}\left(c_{i} d_{i}\right)^{n-i} \cdot x^{M},
\end{aligned}
$$


with $M$ specified below; whence we get:

$$
\left\{\begin{array}{l}
W\left(\exp \left(c_{1} x^{d_{1}}\right), \ldots, \exp \left(c_{m} x^{d_{m}}\right), \exp \left(c_{m+1} x^{d_{m}}\right), \ldots, \exp \left(c_{n} x^{d_{m}}\right)\right) \sim \\
\quad \sim K \cdot x^{M} \cdot \exp \left(\sum_{i=1}^{n} c_{i} x^{d_{i}}\right), x \rightarrow+\infty
\end{array}\right.
$$

where:

$$
\begin{gathered}
K \equiv K\left(n, m, c_{i}, d_{i}\right):=(-1)^{n(n-1) / 2} \cdot V\left(c_{n}, c_{n-1}, \ldots, c_{m+1}\right) \times \\
\times\left(d_{m}\right)^{(n-m)(n-m-1) / 2} \cdot \prod_{i=1}^{m}\left(c_{i} d_{i}\right)^{n-i} \\
M \equiv M\left(n, m, d_{i}\right):=\left(d_{m}-1\right)(n-m)(n-m-1) / 2+\left(d_{1}-1\right)(n-1)+ \\
+\left(d_{2}-1\right)(n-2)+\cdots+\left(d_{m}-1\right)(n-m) .
\end{gathered}
$$

Example 5.6. A "power-logarithmic-exponential" asymptotic scale. (i) For the scale

$\left\{\begin{array}{l}x^{\alpha_{1}}(\log x)^{b_{1}} \exp \left(c_{1} x^{d_{1}}\right) \gg x^{\alpha_{2}}(\log x)^{b_{2}} \exp \left(c_{2} x^{d_{2}}\right) \gg \cdots \gg x^{\alpha_{n}}(\log x)^{b_{n}} \exp \left(c_{n} x^{d_{n}}\right), x \rightarrow+\infty \\ \alpha_{i}, b_{i} \in \mathbb{R} ; c_{i}, d_{i} \text { satisfy one of the set of conditions }(162),(163) \text { or }(166)\end{array}\right.$

the sole difference with respect to example 5.4 is that we have the asymptotic relation $\phi_{i}^{\prime}(x) / \phi_{i}(x) \sim$ $c_{i} d_{i} x^{d_{i}-1}$ instead of equality; but this is immaterial in estimating $V\left(\frac{\phi_{1}^{\prime}(x)}{\phi_{1}(x)}, \ldots, \frac{\phi_{n}^{\prime}(x)}{\phi_{n}(x)}\right)$. For instance, under conditions in (162) or in (163), in formula (164) only the exponential factor has to be replaced by the product of the present functions $\phi_{i}$ and we get the asymptotic relation:

$$
\left\{\begin{array}{l}
W\left(x^{\alpha_{1}}(\log x)^{b_{1}} \exp \left(c_{1} x^{d_{1}}\right), \ldots, x^{\alpha_{n}}(\log x)^{b_{n}} \exp \left(c_{n} x^{d_{n}}\right)\right) \sim \\
\sim(-1)^{n(n-1) / 2}\left(\prod_{i=1}^{n-1}\left(c_{i} d_{i}\right)^{n-i}\right) \cdot x^{M+\alpha_{1}+\cdots+\alpha_{n}} \cdot(\log x)^{b_{1}+\cdots+b_{n}} \cdot \exp \left(\sum_{i=1}^{n} c_{i} x^{d_{i}}\right),
\end{array}\right.
$$

where $M$ is defined in (165). Analogous situations under conditions in (166).

(ii) The case of coinciding $d_{i}$. For the scale

$$
\left\{\begin{array}{l}
x^{\alpha_{1}}(\log x)^{b_{1}} \exp \left(c_{1} x^{d}\right) \gg \cdots \gg x^{\alpha_{n}}(\log x)^{b_{n}} \exp \left(c_{n} x^{d}\right), x \rightarrow+\infty \\
\alpha_{i}, b_{i} \in \mathbb{R} ; \quad d>0, \quad c_{1}>\cdots>c_{n}, c_{i} \neq 0
\end{array}\right.
$$

we have relation:

$$
\begin{aligned}
& W\left(x^{\alpha_{1}}(\log x)^{b_{1}} \exp \left(c_{1} x^{d}\right), \ldots, x^{\alpha_{n}}(\log x)^{b_{n}} \exp \left(c_{n} x^{d}\right)\right) \sim V\left(c_{1}, \ldots, c_{n}\right) \cdot d^{n(n-1) / 2} \times \\
& \times x^{(d-1)[n(n-1) / 2]+\alpha_{1}+\cdots+\alpha_{n}} \cdot(\log x)^{b_{1}+\cdots+b_{n}} \cdot \exp \left[\left(c_{1}+\cdots+c_{n}\right) x^{d}\right] .
\end{aligned}
$$

The case $d_{i}=1$ is reported in the next example.

Example 5.7. "Power-logarithmic-simple exponential" asymptotic scale. For the scale

$$
\left\{\begin{array}{l}
x^{\alpha_{1}}(\log x)^{b_{1}} e^{c_{1} x} \gg x^{\alpha_{2}}(\log x)^{b_{2}} e^{c_{2} x} \gg \cdots \gg x^{\alpha_{n}}(\log x)^{b_{n}} e^{c_{n} x}, x \rightarrow+\infty \\
\alpha_{i}, b_{i} \in \mathbb{R} ; c_{1}>c_{2}>\cdots>c_{n} ; c_{i} \neq 0
\end{array}\right.
$$

we have the asymptotic relation:

$$
\left\{\begin{array}{l}
W\left(x^{\alpha_{1}}(\log x)^{b_{1}} e^{c_{1} x}, \ldots, x^{\alpha_{n}}(\log x)^{b_{n}} e^{c_{n} x}\right) \sim \\
\sim V\left(c_{1}, \ldots, c_{n}\right) \cdot x^{\alpha_{1}+\alpha_{2}+\cdots+\alpha_{n}} \cdot(\log x)^{b_{1}+b_{2}+\cdots+b_{n}} \cdot e^{\left(c_{1}+c_{2} \cdots+c_{n}\right) x}, x \rightarrow+\infty
\end{array}\right.
$$

Example 5.8. An "iterated-exponentials" asymptotic scale. The $n$-tuple of functions 


$$
\left\{\begin{array}{l}
\left(\exp _{p_{1}}(x)\right)^{b_{1}} \cdot\left(\exp _{q_{1}}(x)\right)^{c_{1}} \cdot R_{\alpha_{1}}(x), \ldots,\left(\exp _{p_{n}}(x)\right)^{b_{n}} \cdot\left(\exp _{q_{n}}(x)\right)^{c_{n}} \cdot R_{\alpha_{n}}(x), \\
R_{\alpha_{i}} \text { smoothly varying at }+\infty \text { of order } n-1 \text { and of index } \alpha_{i}, \\
b_{i} \in \mathbb{R} \backslash\{0\} ; c_{i} \in \mathbb{R} ; p_{1}>\cdots>p_{n} \geq 1 ; 1 \leq q_{i}<p_{i} \forall i
\end{array}\right.
$$

as well as their logariyhmic derivatives can be rearranged so as to form asymptotic scales at $+\infty$ and:

$$
\begin{aligned}
& D_{\ell}\left[\left(\exp _{p_{i}}(x)\right)^{b_{i}} \cdot\left(\exp _{q_{i}}(x)\right)^{c_{i}} \cdot R_{\alpha_{i}}(x)\right]= \\
& =b_{i} \cdot D \exp _{p_{i}-1}(x)+c_{i} \cdot D \exp _{q_{i}-1}(x)+x^{-1}\left[\alpha_{i}+o(1)\right] \sim b_{i} \prod_{k=0}^{p_{i}-1} \exp _{k}(x), x \rightarrow+\infty
\end{aligned}
$$

(In the range of the index in the product we prefer to include the value $k=0$, though $\exp _{0}(x) \equiv 1$, to avoid some misunderstanding in the case $p_{i}=1$.)

Hence we have the asymptotic formula inferred from (140):

$$
\left\{\begin{array}{l}
W\left(\left(\exp _{p_{1}}(x)\right)^{b_{1}} \cdot\left(\exp _{q_{1}}(x)\right)^{c_{1}} \cdot R_{\alpha_{1}}(x), \ldots,\left(\exp _{p_{n}}(x)\right)^{b_{n}} \cdot\left(\exp _{q_{n}}(x)\right)^{c_{n}} \cdot R_{\alpha_{n}}(x)\right) \sim \\
\sim(-1)^{n(n-1) / 2} \cdot\left(\prod_{i=1}^{n-1}\left(b_{i}\right)^{n-i}\right) \cdot\left(\prod_{i=1}^{n}\left(\exp _{p_{i}}(x)\right)^{b_{i}} \cdot\left(\exp _{q_{i}}(x)\right)^{c_{i}} \cdot R_{\alpha_{i}}(x)\right) \times \\
\times \prod_{i=1}^{n-1}\left(\prod_{k=0}^{p_{i}-1} \exp _{k}(x)\right)^{n-i}, x \rightarrow+\infty .
\end{array}\right.
$$

If the restrictions on the $b_{i}$ 's and $p_{i}$ 's in (184) are replaced by

$$
b_{i} \neq 0 ; \quad p_{1} \geq \cdots \geq p_{n} \geq 1 ; \quad p_{i}=p_{j} \Rightarrow b_{i} \neq b_{j},
$$

the same asymptotic formula (185) holds true with the sole difference that the constant factor $(-1)^{n(n-1) / 2}$ is replaced by a suitable nonzero constant $C$ solely dependent on the $b_{i}$ 's; hence we have:

$$
\left\{\begin{array}{l}
W\left(\left(\exp _{p_{1}}(x)\right)^{b_{1}} \cdot\left(\exp _{q_{1}}(x)\right)^{c_{1}} \cdot R_{1}(x), \ldots,\left(\exp _{p_{n}}(x)\right)^{b_{n}} \cdot\left(\exp _{q_{n}}(x)\right)^{c_{n}} \cdot R_{n}(x)\right) \sim \\
\sim C \cdot\left(\prod_{i=1}^{n}\left(\exp _{p_{i}}(x)\right)^{b_{i}} \cdot\left(\exp _{q_{i}}(x)\right)^{c_{i}} \cdot R_{i}(x)\right) \cdot \prod_{i=1}^{n-1}\left(\prod_{k=0}^{p_{i}-1} \exp _{k}(x)\right)^{n-i}, x \rightarrow+\infty
\end{array}\right.
$$

Example 5.9. An asymptotic scale with exponentials and one constant. If in a scale containing only functions of type $\exp \left(c_{i} x^{d_{i}}\right)$ one of the $c_{i}$ 's is zero then the corresponding function is the constant " 1 " and we may have resort to formula (148). For instance, for each of the scales:

$$
\begin{aligned}
& \exp \left(c_{1} x^{d_{1}}\right) \gg \exp \left(c_{2} x^{d_{2}}\right) \gg \cdots \gg \exp \left(c_{n} x^{d_{n}}\right) \gg 1,\left(c_{i}>0 ; d_{1}>d_{2}>\cdots>d_{n}>0\right), \\
& 1 \gg \exp \left(c_{1} x^{d_{1}}\right) \gg \exp \left(c_{2} x^{d_{2}}\right) \gg \cdots \gg \exp \left(c_{n} x^{d_{n}}\right),\left(c_{i}<0 ; 0<d_{1}<d_{2}<\cdots<d_{n}\right),
\end{aligned}
$$

we have:

$$
\begin{aligned}
& W\left(1, \exp \left(c_{1} x^{d_{1}}\right), \ldots, \exp \left(c_{n} x^{d_{n}}\right)\right)=W\left(c_{1} d_{1} x^{d_{1}-1} \exp \left(c_{1} x^{d_{1}}\right), \ldots, c_{n} d_{n} x^{d_{n}-1} \exp \left(c_{n} x^{d_{n}}\right)\right)= \\
& =\left(\prod_{i=1}^{n} c_{i} d_{i}\right) \cdot W\left(x^{d_{1}-1} \exp \left(c_{1} x^{d_{1}}\right), \ldots, x^{d_{n}-1} \exp \left(c_{n} x^{d_{n}}\right)\right) \stackrel{(169)}{\sim} \\
& \sim(-1)^{n(n-1) / 2}\left(\prod_{i=1}^{n} c_{i} d_{i}\right)\left(\prod_{i=1}^{n-1}\left(c_{i} d_{i}\right)^{n-i}\right) \cdot x^{M+\alpha_{1}+\cdots+\alpha_{n}} \cdot \exp \left(\sum_{i=1}^{n} c_{i} x^{d_{i}}\right),
\end{aligned}
$$

where $M$ is defined in (165). 


\section{Applications to Asymptotic Expansions}

Before doing meaningful applications of the previous formulas we recall the framework wherein we constructed an analytic theory of finite asymptotic expansions in the real domain.

(A) Assumptions. Let the ordered n-tuple of real-valued functions $\left(\phi_{1}, \ldots, \phi_{n}\right), n \geq 2$, satisfy the following properties:

$$
\begin{gathered}
\phi_{i} \in A C^{n-1}[T,+\infty[, 1 \leq i \leq n ; \\
\phi_{i}(x) \neq 0 \text { on }[T,+\infty[, 1 \leq i \leq n ; \\
\phi_{1}(x) \gg \phi_{2}(x) \gg \cdots \gg \phi_{n}(x), x \rightarrow+\infty ; \\
W\left(\phi_{1}(x), \ldots, \phi_{i}(x)\right) \neq 0 \text { on }[T,+\infty[, 1 \leq i \leq n .
\end{gathered}
$$

In [3; Def.2.1, p. 459, and Prop. 2.3, p. 460] $\left(\phi_{1}, \ldots, \phi_{n}\right)$ is labelled a "Chebyshev asymptotic scale" on the half-open interval $[T,+\infty[$. Moreover, let the operator

$$
L_{\phi_{1}, \ldots, \phi_{n}} u:=W\left(\phi_{1}(x), \ldots, \phi_{n}(x), u\right) / W\left(\phi_{1}(x), \ldots, \phi_{n}(x)\right)
$$

be the unique linear ordinary differential operator of type:

$$
\left\{\begin{array}{l}
L_{n} u:=u^{(n)}+\alpha_{n-1}(x) u^{(n-1)}+\cdots+\alpha_{0}(x) u \quad \forall u \in A C^{n-1}[T,+\infty[ \\
\alpha_{i} \in L_{l o c}^{1}[T,+\infty[, 0 \leq i \leq n-1
\end{array}\right.
$$

acting on the space $A C^{n-1}\left[T,+\infty\left[\right.\right.$ and such that $\operatorname{ker} L_{\phi_{1}, \ldots, \phi_{n}}=\operatorname{span}\left(\phi_{1}, \ldots, \phi_{n}\right)$.

One of the main results in [3; Th. 5.1, p. 469] states that for a function $f \in A C^{n-1}[T,+\infty$ [ the integral condition

$$
\int_{T}^{+\infty} \frac{W\left(\phi_{1}(t), \ldots, \phi_{n-1}(t)\right)}{W\left(\phi_{1}(t), \ldots, \phi_{n}(t)\right)} \cdot L_{\phi_{1}, \ldots, \phi_{n}}[f(t)] \mathrm{d} t \text { convergent }
$$

grants the validity of an asymptotic expansion:

$$
f(x)=a_{1} \phi_{1}(x)+\cdots+a_{n} \phi_{n}(x)+o\left(\phi_{n}(x)\right), x \rightarrow+\infty .
$$

Condition (198) does indeed characterize a set of $n$ asymptotic expansions formed by (199) and $n-1$ expansions formally obtained from (199) by applying suitable linear differential operators of orders $1,2, \ldots, n-1,[3 ;$ p. 469]. Apart from the nontrivial theoretical facts concerning such operators there is a simple constructive procedure to define and visualize them highlighted in [5; Th. 13.1]. Consider the element $u \in \operatorname{span}\left(\phi_{1}, \ldots, \phi_{n}\right)$ defined by $u:=\phi_{1}+\cdots+\phi_{n}$; dividing both sides by $\phi_{1}$ one gets an identity through which an operator is defined:

$$
u / \phi_{1}=1+\left(\phi_{2} / \phi_{1}\right)+\cdots+\left(\phi_{n} / \phi_{1}\right) ; \quad M_{0}(u):=u / \phi_{1} .
$$

We now take derivatives of both sides of the identity and then divide by the first term on the right so getting a new identity through which a first-order differential operator is defined:

$$
\frac{\left(u / \phi_{1}\right)^{\prime}}{\left(\phi_{2} / \phi_{1}\right)^{\prime}}=1+\frac{\left(\phi_{3} / \phi_{1}\right)^{\prime}}{\left(\phi_{2} / \phi_{1}\right)^{\prime}}+\cdots+\frac{\left(\phi_{n} / \phi_{1}\right)^{\prime}}{\left(\phi_{2} / \phi_{1}\right)^{\prime}} ; \quad M_{1}(u):=\frac{\left(u / \phi_{1}\right)^{\prime}}{\left(\phi_{2} / \phi_{1}\right)^{\prime}} .
$$

We repeat the procedure taking derivatives of both sides of the last identity and then dividing by the first term on the right so getting a new identity through which a second-order differential operator is defined:

$$
\begin{aligned}
& \left(\frac{\left(u / \phi_{1}\right)^{\prime}}{\left(\phi_{2} / \phi_{1}\right)^{\prime}}\right)^{\prime} /\left(\frac{\left(\phi_{3} / \phi_{1}\right)^{\prime}}{\left(\phi_{2} / \phi_{1}\right)^{\prime}}\right)^{\prime}=1+\left(\frac{\left(\phi_{4} / \phi_{1}\right)^{\prime}}{\left(\phi_{2} / \phi_{1}\right)^{\prime}}\right)^{\prime} /\left(\frac{\left(\phi_{3} / \phi_{1}\right)^{\prime}}{\left(\phi_{2} / \phi_{1}\right)^{\prime}}\right)^{\prime}+\cdots \\
& \ldots+\left(\frac{\left(\phi_{n} / \phi_{1}\right)^{\prime}}{\left(\phi_{2} / \phi_{1}\right)^{\prime}}\right)^{\prime} /\left(\frac{\left(\phi_{3} / \phi_{1}\right)^{\prime}}{\left(\phi_{2} / \phi_{1}\right)^{\prime}}\right)^{\prime} ; \quad M_{2}(u):=\left(\frac{\left(u / \phi_{1}\right)^{\prime}}{\left(\phi_{2} / \phi_{1}\right)^{\prime}}\right)^{\prime} /\left(\frac{\left(\phi_{3} / \phi_{1}\right)^{\prime}}{\left(\phi_{2} / \phi_{1}\right)^{\prime}}\right)^{\prime} .
\end{aligned}
$$


And the procedure is repeated $n-1$ times. The operators $M_{1}, \ldots, M_{n-1}$ are those for which condition (198) grants the formal applicability to the expansion (199) so yielding $n-1$ more asymptotic expansions:

$$
M_{k}[f(x)]=a_{k+1} M_{k}\left[\phi_{k+1}(x)\right]+\cdots+a_{n} M_{k}\left[\phi_{n}(x)\right]+o\left(M_{k}\left[\phi_{n}(x)\right]\right), x \rightarrow+\infty, 1 \leq k \leq n-1 ;
$$

Notice that the first term in each expansion is lost in the successive expansion, just the same phenomenon as in Taylor's formula. Our theory also yields integral representations of the remainders in (199) and (203); for instance the remainder $R_{0}(x)$ in (199) can be represented as:

$$
R_{0}(x)=\frac{(-1)^{n}}{q_{0}(x)} \int_{x}^{x_{0}} \frac{1}{q_{1}} \cdots \int_{t_{n-2}}^{x_{0}} \frac{1}{q_{n-1}} \int_{t_{n-1}}^{x_{0}} \frac{L_{\phi_{1}, \ldots, \phi_{n}}[f(t)]}{q_{n}(t)} \mathrm{d} t
$$

where the functions $q_{i}$ are defined as follows:

$$
\left\{\begin{array}{l}
q_{0}:=1 /\left|\phi_{1}\right| ; q_{1}:=\left(\phi_{1}\right)^{2} /\left|W\left(\phi_{1}, \phi_{2}\right)\right| ; \\
q_{i}:=\left[W\left(\phi_{1}, \ldots, \phi_{i}\right)\right]^{2} /\left|W\left(\phi_{1}, \ldots, \phi_{i-1}\right) \cdot W\left(\phi_{1}, \ldots, \phi_{i+1}\right)\right|, 2 \leq i \leq n-1 ; \\
q_{n}:=\left|q_{0} q_{1} \ldots q_{n-1}\right|^{-1} \equiv\left|W\left(\phi_{1}, \ldots, \phi_{n}\right) / W\left(\phi_{1}, \ldots, \phi_{n-1}\right)\right| .
\end{array}\right.
$$

Notice that the aim of the theory, as developed by us, does not consist in expressing the integral in (204a) as the quantity " $f(x)-\sum_{i=1}^{n} a_{i} \phi_{i}(x)$ "; on the contrary the integral condition is used as a sufficient condition to grant (199) (with suitable coefficients $a_{i}$ ); and it is not a rough sufficient condition in so far as it is also necessary whenever certain additional formal differentiated expansions hold true. The core of the whole theory in [3] is just the problem of formal differentiation of finite asymptotic expansions highlighting that, under assumptions (192)-(195), there are at least two different $(n-1)$-tuples of differential operators of orders $1,2, \ldots, n-1$ which can be formally applied to (199). These two circumstances are characterized: one of them by the (perhaps conditional) convergence of the improper integral in (198) and the other by the convergence of a certain iterated improper integral: see $[3 ; \S 4]$. This happens even in the most elementary case of an asymptotic straight line " $f(x)=a x+b+o(1), x \rightarrow+\infty$," in which case the two different operators are $u^{\prime}$ and $\left(x^{-1} u\right)^{\prime}$. A particularly meaningful special case is that of a function $f$ satisfying the differential inequality

$$
L_{\phi_{1}, \ldots, \phi_{n}}[f(x)] \geq 0 \text { a. e. on }[T,+\infty[\text {. }
$$

Under this special assumption (198) characterizes the sole expansion (199) which turns out to be automatically formally differentiable $(n-1)$ times according to both the pertinent $(n-1)$-tuples of differential operators, namely: the above-constructed $M_{k}$ 's and the $L_{k}$ 's constructed by a similar procedure starting from $u=\phi_{1}+\cdots+\phi_{n}$ and dividing both sides by the last term on the right before taking derivatives at each step.

(B) Sufficient conditions involving absolute convergence.

Coming back to the subject of the present paper, if one succeeds in evaluating the exact asymptotic behavior of the Wronskians in (198) then one can characterize the absolute convergence of the integral so getting an applicable sufficient condition for (199) to hold. We collect in the following theorem results of this type directly inferred from Theorems 9-10 for various classes of scales.

Theorem 11. (I) Smoothly-varying scales. Let the functions $\phi_{i}$ satisfy conditions (192) to (195) and moreover

$$
\phi_{i}^{(k)}(x) / \phi_{i}(x)=x^{-k}\left[\left(\alpha_{i}\right)^{\underline{k}}+o\left(x^{-k}\right)\right], x \rightarrow+\infty ; 1 \leq i \leq n, 1 \leq k \leq n-1,
$$


where $\alpha_{1}>\cdots>\alpha_{n}$. Then absolute convergence of the integral in (198) is granted by

$$
\int_{T}^{+\infty} t^{n-1}\left|\phi_{n}(t)\right|^{-1} \cdot\left|L_{\phi_{1}, \ldots, \phi_{n}}[f(t)]\right| \mathrm{d} t \text { convergent. }
$$

(II) Rapidly-varying scales. Let the functions $\phi_{i}$ satisfy conditions (192) to (195), (135) and moreover:

$$
\frac{\phi_{1}^{\prime}(x)}{\phi_{1}(x)} \gg \cdots \gg \frac{\phi_{n}^{\prime}(x)}{\phi_{n}(x)}, x \rightarrow+\infty .
$$

Then absolute convergence of the integral in (198) is granted by

$$
\int_{T}^{+\infty}\left|\prod_{i=1}^{n-1}\left(\frac{\phi_{i}^{\prime}(t)}{\phi_{i}(t)}\right)\right|^{-1} \cdot\left|\phi_{n}(t)\right|^{-1} \cdot L_{\phi_{1}, \ldots, \phi_{n}}[f(t)] \mid \mathrm{d} t \text { convergent. }
$$

(III) Rapidly-varying scales with proportional logarithmic derivatives. Let the functions $\phi_{i}$ satisfy conditions (192) to (195), (135) and moreover:

$$
\phi_{i}^{\prime}(x) / \phi_{i}(x)=c_{i} \phi(x)+o(\phi(x)), x \rightarrow+\infty ; 1 \leq i \leq n ; c_{1}>\cdots>c_{n} .
$$

Whenever $\phi(x) \equiv 1$ the $\phi_{i}$ 's are sometimes termed exponentially-varying at $+\infty:[7 ; \S 8]$. Then absolute convergence of the integral in (198) is granted by

$$
\int_{T}^{+\infty}|\phi(t)|^{1-n} \cdot\left|\phi_{n}(t)\right|^{-1} \cdot\left|L_{\phi_{1}, \ldots, \phi_{n}}[f(t)]\right| \mathrm{d} t \text { convergent. }
$$

which is the form assumed by (208) in the present case. (In condition (205) the role of $\phi(x)$ is played by $x^{-1}$.)

(IV) Mixed rapidly-varying scales of the two previous types. Let the functions $\phi_{i}$ satisfy conditions (192) to (195), (135) and moreover let them be grouped in ordered $i_{k}$-tuples satisfying (145)-(146). Then absolute convergence of the integral in (198) is granted by the same condition (208) wherein each group of asymptotically-equivalent factors $\left(\phi_{i}^{\prime}(t) / \phi_{i}(t)\right)^{-1}$ may be replaced by only one factor with the appropriate exponent.

\section{(C) Special scales admitting of explicit characterizations.}

For the majority of scales encountered in applications when studying asymptotics of non-oscillatory differentiable functions it is easy to see that the sole principal parts of the Wronskians in (198) suffice to establish the convergence - perhaps conditional - or nor of the integral in (198). Here we have in mind those comparison functions $\phi_{i} \in C^{\infty}[T,+\infty$ [ which can be represented by products of compositions of a finite number of logarithms, powers and exponentials. The Wronskians of such functions and their derivatives, if not $\equiv 0$, admit of similar representations hence these Wronskians do not vanish and are monotonic on a neighborhood of $+\infty$ and we may have resort to the following corollary of the classical Abel's test for convergence of improper integrals.

Lemma 12. If

$$
\left\{\begin{array}{l}
f \in L_{l o c}^{1}[T,+\infty[; \phi, \psi \in A C[T,+\infty[ \\
\psi(x)=o(1), x \rightarrow+\infty ; \psi^{\prime} \text { either } \leq 0 \text { a. e. or } \geq 0 \text { a.e.; }
\end{array}\right.
$$

then:

$$
\int_{T}^{+\infty} \phi \cdot f \text { converges } \Longleftrightarrow \int_{T}^{+\infty}(\phi+\phi \psi) \cdot f \text { converges. }
$$

(Here $\phi$ stands for the principal part at $+\infty$ of the function $\phi+\phi \psi$.) 
Proof. Abel's test (simply proved by a suitable integration by parts) states that

$$
\int_{T}^{+\infty} f \text { convergent } \Rightarrow \int_{T}^{+\infty} \psi \cdot f \text { convergent }
$$

under the above assumptions on $f, \psi$. In our lemma, if $\int_{T}^{+\infty} \phi f$ converges then:

$$
\int_{T}^{+\infty}(\phi+\phi \psi) \cdot f=\int_{T}^{+\infty} \phi \cdot f+\int_{T}^{+\infty} \psi \cdot(\phi \cdot f) \text { convergent by }(213) ;
$$

and viceversa, if $\int_{T}^{+\infty}(\phi+\phi \psi) f$ converges then for $T_{0}$ large enough we have:

$$
\int_{T_{0}}^{+\infty} \phi \cdot f=\int_{T_{0}}^{+\infty}(\phi+\phi \psi) \cdot f-\int_{T_{0}}^{+\infty} \frac{\phi \psi}{\phi+\phi \psi}(\phi+\phi \psi) \cdot f \text { convergent by (213) }
$$

as $\phi \psi /(\phi+\phi \psi)=\psi /(1+\psi)$ and $D(\psi /(1+\psi))=\psi^{\prime} /(1+\psi)^{2}$ so that the function $\psi /(1+\psi)$ satisfies the same assumptions than $\psi$ on a suitable neighborhood of $+\infty$.

In all of the following examples the comparison functions $\phi_{i}$ are of class $C^{\infty}[T,+\infty$ [ for some suitable $T$, satisfy conditions (192) to (195) and their Wronskians, ratios of Wronskians and pertinent derivatives are ultimately strictly monotonic so that Lemma 12 can be applied and we may assert that the integral in (198) converges iff the corresponding integral converges wherein only the principal part at $+\infty$ of the ratio of the Wronskians appears. Referring to the examples in $\S 5$ we omit the case of a general real-power asymptotic scale as it has been thoroughly studied in $[5 ; \S 15]$.

Warning. In each of the following integral conditions the symbol $L$ has a different meaning as it stands for the operator $L_{\phi_{1}, \ldots, \phi_{n}}$ defined in (196)-(197) in relation to the specific scale at hand $\left(\phi_{1}, \ldots, \phi_{n}\right)$. Do not marvel if not all the functions in the given scale explicitly appear in the integral conditions: they implicitly appear in the quantity $L[f(t)]$. Formal differentiability is meant in the sense explained at the outset of the present section.

1. "Power-logarithmic" asymptotic expansions. (I) A function $f \in A C^{n-1}[T,+\infty[$ admits of an asymptotic expansion of type

$$
\left\{\begin{array}{l}
f(x)=a_{1} x^{\alpha_{1}}(\log x)^{\beta_{1}}+\cdots+a_{n} x^{\alpha_{n}}(\log x)^{\beta_{n}}+o\left(x^{\alpha_{n}}(\log x)^{\beta_{n}}\right), x \rightarrow+\infty \\
\alpha_{1}>\alpha_{2}>\cdots>\alpha_{n} ; \beta_{i} \in \mathbb{R}
\end{array}\right.
$$

formally differentiable $n-1$ times, iff

$$
\int^{+\infty} t^{n-1-\alpha_{n}} \cdot(\log x)^{-\beta_{n}} \cdot L[f(t)] \mathrm{d} t \quad \text { converges. }
$$

(II) Similarly, an asymptotic expansion of type

$$
\left\{\begin{array}{l}
f(x)=a_{1} x^{\alpha_{1}} \mathcal{L}_{1}(x)+\cdots+a_{n} x^{\alpha_{n}} \mathcal{L}_{n}(x)+o\left(x^{\alpha_{n}} L_{n}(x)\right), x \rightarrow+\infty \\
\alpha_{1}>\alpha_{2}>\cdots>\alpha_{n} ; \mathcal{L}_{i} \text { defined in }(159)
\end{array}\right.
$$

formally differentiable $n-1$ times, holds true iff

$$
\int^{+\infty} t^{n-1-\alpha_{n}} \cdot\left(\mathcal{L}_{n}(t)\right)^{-1} \cdot L[f(t)] \mathrm{d} t \quad \text { converges. }
$$


2. "Power-logarithmic-exponential" asymptotic expansions. (I) A function $f \in A C^{n-1}[T,+\infty[$ admits of an asymptotic expansion of type

$$
\left\{\begin{aligned}
& f(x)= a_{1} x^{\alpha_{1}}(\log x)^{b_{1}} \exp \left(c_{1} x^{d_{1}}\right)+\cdots+a_{n} x^{\alpha_{n}}(\log x)^{b_{n}} \exp \left(c_{n} x^{d_{n}}\right)+ \\
&+o\left(x^{\alpha_{n}}(\log x)^{b_{n}} \exp \left(c_{n} x^{d_{n}}\right)\right), x \rightarrow+\infty \\
& \alpha_{i}, b_{i} \in \mathbb{R} ; c_{i}, d_{i} \text { satisfy one of the conditions (162), (163) or (166) }
\end{aligned}\right.
$$

formally differentiable $n-1$ times, iff

$$
\int^{+\infty} t^{n-1-\alpha_{n}-d_{1}-\cdots-d_{n-1}} \cdot(\log x)^{-b_{n}} \cdot \exp \left(-c_{n} x^{d_{n}}\right) \cdot L[f(t)] \mathrm{d} t \quad \text { converges. }
$$

(II) For the special case of coinciding exponents, $d_{i}=d \forall i$ and $c_{1}>\cdots>c_{n}, c_{i} \neq 0$, the integral condition becomes:

$$
\int^{+\infty} t^{(n-1)(1-d)-\alpha_{n}} \cdot(\log x)^{-b_{n}} \cdot \exp \left(-c_{n} x^{d}\right) \cdot L[f(t)] \mathrm{d} t \quad \text { converges. }
$$

3. Asymptotic expansions with simple exponentials. A function $f \in A C^{n-1}[T,+\infty[$ admits of an asymptotic expansion of type

$\left\{\begin{array}{l}f(x)=a_{1} x^{\alpha_{1}}(\log x)^{b_{1}} e^{c_{1} x}+\cdots+a_{n} x^{\alpha_{n}}(\log x)^{b_{n}} e^{c_{n} x}+o\left(x^{\alpha_{n}}(\log x)^{b_{n}} e^{c_{n} x}\right), x \rightarrow+\infty, \\ \alpha_{i}, b_{i} \in \mathbb{R} ; c_{1}>c_{2}>\cdots>c_{n} ; c_{i} \neq 0 ;\end{array}\right.$

formally differentiable $n-1$ times, iff

$$
\int^{+\infty} t^{-\alpha_{n}} \cdot(\log x)^{-b_{n}} \cdot e^{-c_{n} x} \cdot L[f(t)] \mathrm{d} t \quad \text { converges. }
$$

4. Asymptotic expansions with iterated exponentials. (I) A function $f \in A C^{n-1}[T,+\infty[$ admits of an asymptotic expansion of type

$$
\left\{\begin{array}{l}
f(x)=a_{1}\left(\exp _{p_{1}}(x)\right)^{b_{1}} \cdot\left(\exp _{q_{1}}(x)\right)^{c_{1}} \cdot x^{\alpha_{1}}+\cdots+a_{n}\left(\exp _{p_{n}}(x)\right)^{b_{n}}\left(\exp _{q_{n}}(x)\right)^{c_{n}} \cdot x^{\alpha_{n}}+ \\
+o\left(\left(\exp _{p_{n}}(x)\right)^{b_{n}}\left(\exp _{q_{n}}(x)\right)^{c_{n}} \cdot x^{\alpha_{n}}\right), x \rightarrow+\infty ; \\
\text { either }: \quad b_{i}>0 \forall i ; c_{i}, \alpha_{i} \in \mathbb{R} ; p_{1} \geq \cdots \geq p_{n} \geq 1 ; p_{i}=p_{j} \Rightarrow b_{i}>b_{j} ; 0 \leq q_{i}<p_{i} \forall i ; \\
\text { or }: \quad b_{i}<0 \forall i ; c_{i}, \alpha_{i} \in \mathbb{R} ; 1 \leq p_{1} \leq \cdots \leq p_{n} ; p_{i}=p_{j} \Rightarrow b_{i}>b_{j} ; 0 \leq q_{i}<p_{i} \forall i ;
\end{array}\right.
$$

formally differentiable $n-1$ times, iff

$$
\int^{+\infty}\left[\left(\exp _{p_{n}}(t)\right)^{b_{n}} \cdot\left(\exp _{q_{n}}(t)\right)^{c_{n}} \cdot t^{\alpha_{n}}\right]^{-1} \cdot\left(\prod_{k=0}^{p_{n-1}-1} \exp _{k}(x)\right)^{-1} \cdot L[f(t)] \mathrm{d} t \text { converges. }
$$

(II) For the special case of the expansion:

$$
\left\{\begin{array}{l}
f(x)=a_{1}\left(\exp _{n}(x)\right)^{b_{1}} \cdot x^{\alpha_{1}}+\cdots+a_{n}\left(\exp _{1}(x)\right)^{b_{n}} \cdot x^{\alpha_{n}}+ \\
+o\left(\left(\exp _{1}(x)\right)^{b_{n}} \cdot x^{\alpha_{n}}\right), x \rightarrow+\infty, \quad\left(b_{i}>0 \forall i\right)
\end{array}\right.
$$

the integral condition becomes:

$$
\int^{+\infty} t^{-\alpha_{n}} \cdot \exp \left(\left(-b_{n}-1\right) t\right) \cdot L[f(t)] \mathrm{d} t \quad \text { converges. }
$$




\section{Historical and Bibliographical Notes}

- (Wronskian identities).The identity (25) is reported in Pólya and Szëgo [8; Part VII, no. 57, p. $108]$ and it could be called Hesse-Christoffel's identity according to the historical notes by Krusemeyer [9; p. 47] who reports an elementary proof by suitable row/column manipulations.

- The identity (26), trivially true for $n=2$, is reported in Pólya and Szëgo as well: [8; Part VII, no. 56]. It is also proved by simple row/column manipulations. It seems to have been discovered by Mina [10; p. 198]. A matricial proof based on Faà di Bruno's formula for higher derivatives of compositions has ben given by Chu Wenchang [11] together with many examples and extensions.

- (About Proposition 3). Proposition 3 is taken from Proposition 4.1 in [6; pp. 807-808] wherein formula (4.12) has been preceded by an erroneous statement. Formula (4.13) in [6], which is formula (52) in the present paper, is correct. The last five lines in the statement of Proposition 4.1 in [6] must be replaced by the following ones:

... Relations in (4.10) do not imply the asymptotic scale:

$$
f^{(n+1)}(x) \gg f^{(n)}(x) \gg \cdots \gg f^{\prime}(x) \gg f(x), x \rightarrow+\infty,
$$

which characterizes a subclass of rapidly-varying functions. However, a different way of writing relations in (4.6) yields the asymptotic scale:

$$
f^{(n)}(x) \gg x^{-1} f^{(n-1)}(x) \gg x^{-2} f^{(n-2)}(x) \gg \cdots \gg x^{-(n-1)} f^{\prime}(x) \gg x^{-n} f(x), x \rightarrow+\infty .
$$

The mentioned subclass is defined in [7; Def. 8.2, pp. 839-840].

- (Vandermondians). A possible classical reference for formula (58) is Satake [12; p. 61 and p. 68]; more recently a combinatorial proof has been given by Benjamin and Dresden [13]. The special formulas (70)-(71) appear here and there in the literature and sometimes with complicated proofs whereas they directly follow from Hesse-Christoffel's formula. Nonsimple proofs based on Faà di Bruno's formula may be found in Fel'dman [14; pp. 260-262] and in Baker [15; pp. 161-162], whereas sketched proofs are in Van der Poorten [16] and in Zeitlin [17] with some examples. Formula (69) with $g(x) \equiv x$ appears with an elementary proof in Knebelman [18; p. 252] and in Bostan and Dumas [19; p. 723]; it is proved in Güichal [20; p. 68] by the indirect use of differential equations and in Utz [21] by the immediate recourse to Hesse-Christoffel's formula. A simple proof also appears in a note by Shiro and Hirano [22].

- (Asymptotics of Wronskians). We can mention only one result in the literature related to the results in $\S 4$, namely a result on the asymptotic behavior of

$$
W\left(t^{a_{1}}+O\left(t^{a_{1}+1}\right), \ldots, t^{a_{n}}+O\left(t^{a_{n}+1}\right)\right), \text { as } t \rightarrow 0,
$$

obtained by Güichal [20; Prop. 4, p. 67] by a quite different procedure. Other papers may be found in the literature that contain the words "growth order of Wronskians" in their titles, but they are concerned with certain properties of holomorphic functions unrelated to our study.

- (About Lemma 8). The idea underlying Lemma 8 may be traced back to the classic work by Hardy [23; Th. 23, p. 34], the foundation of modern "Asymptotic Analysis". This result by Hardy as well as a slightly more general result in Bourbaki [24; Prop. 12, p.V.9] also highlight asymptotic relations between the powers of $f$ and $g$. If a name has to be attached to Lemma 8 and its variants a fair choice would be "L'Hospital-Hardy's rule" instead of "Charlie's rule" [25] as it seems to be known by some computer scientists. 


\section{References}

[1] A. Granata, Analytic theory of finite asymptotic expansions in the real domain. Part I: two-term expansions of differentiable functions, Analysis Mathematica. 37(4) (2011) 245-287.

[2] A. Granata, The factorizational theory of finite asymptotic expansions in the real domain: a survey of the main results, Advances in Pure Mathematics. 5 (2015) 1-20.

[3] A. Granata, Analytic theory of finite asymptotic expansions in the real domain. Part II-A: the factorizational theory for Chebyshev asymptotic scales, Advances in Pure Mathematics. 5 (2015) 454-480.

[4] A. Granata, Analytic theory of finite asymptotic expansions in the real domain. Part II-B: solutions of differential inequalities and asymptotic admissibility of standard derivatives, Advances in Pure Mathematics. 5 (2015) 481-502.

[5] A. Granata, Analytic theory of finite asymptotic expansions in the real domain. Part II-C: constructive algorithms for canonical factorizations and a special class of asymptotic scales, Advances in Pure Mathematics. 5 (2015) 503-526.

[6] A. Granata, The theory of higher-order types of asymptotic variation for differentiable functions. Part I: higher-order regular, smooth and rapid variation, Advances in Pure Mathematics. 6 (2016) 776-816.

[7] A. Granata, The theory of higher-order types of asymptotic variation for differentiable functions. Part II: algebraic operations and types of exponential variation, Advances in Pure Mathematics. 6 (2016) 817-867.

[8] G. Pólya, G. Szëgo, Problems and theorems in analysis, Vol. II, Springer-Verlag, Berlin, 1976.

[9] M. Krusemeyer, Why does the Wronskian work?, American Mathematical Monthly. 95 (1988) 46-49.

[10] L. Mina, Formule generali delle derivate successive d'una funzione, espresse mediante quelle della sua inversa, Giornale di Matematiche. 43 (1904) 196-212.

[11] C. Wenchang, The Faà di Bruno formula and determinant identities, Linear and Multilinear Algebra. 54(1) (2006) 1-25.

[12] I. Satake, Linear algebra, Marcel Dekker, Inc., New York, 1975.

[13] A.T. Benjamin, G.P. Dresden, A combinatorial proof of Vandermonde's determinant, American Mathematical Monthly. 114 (2007) 338-341.

[14] N.I. Fel'dman, Approximation of certain transcendental numbers. II: The approximation of certain numbers associated with the Weierstrass $\wp$-function, Izvestiya Akademii Nauk SSSR. Seriya Matematicheskaya.15 (1951) 153-176. Translated in: American Mathematical Society Translations, Series 2. 59 (1966) 246-270.

[15] A. Baker, On the periods of the Weierstrass $\wp$-function, Symposia Mathematica, Vol. IV, INDAM, Rome, 1968/69, Academic Press, London, 1970, pp. 155-174.

[16] A. J. Van der Poorten, Some determinants that should be better known, Journal of the Australian Mathematical Society (Series A). 21(03) (1976) 278-288.

[17] D. Zeitlin, A Wronskian, American Mathematical Monthly. 65 (1958) 345-349.

[18] M.S. Knebelman, The Wronskian for linear differential equations, American Mathematical Monthly. 56 (1949) 252-254. 
[19] A. Bostan, Ph. Dumas, Wronskians and linear independence, American Mathematical Monthly. 117 (2010) 722-727.

[20] E.N. Güichal, A relation between Gram and Wronsky determinants, Linear Algebra and its Applications. 72 (1985) 59-72.

[21] W.R. Utz, An application of the Wronskian, American Mathematical Monthly. 76 (1969) 56-57.

[22] A. Shiro, T. Hirano, On the proof of a formula of Wronski, Sûgaku. 29 (1977/78) 364-365.

[23] G.H. Hardy, Orders of infinity. The 'Infinitärcalcül' of Paul du Bois-Reymond, Cambridge the University Press, 1924.

[24] N. Bourbaki, Fonctions d'une variable Réelle-Théorie Élémentaire. Hermann, Paris, 1976.

[25] C. Obimbo, A new method to order functions by asymptotic growth rates, Unpublished. Available: https://www.cs.ubc.ca/wccce/Program03/papers/Obi2.pdf. 